\title{
FACTORS AFFECTING RESPONSE TO RECURRENT GENOMIC SELECTION IN SOYBEANS
}

\section{1) Vishnu Ramasubramanian}

1525, Agronomy Hall, ISU

Ames, IA 50011

Phone: 5155986376

Email: vishnur@iastate.edu

\section{2) William Beavis*}

1208, Agronomy Hall, ISU

Ames, IA 50011

Phone: 8004504854 (4412)

Fax: 515294730

E-mail: wdbeavis@ iastate.edu 


\section{Vishnu Ramasubramanian* ${ }^{\dagger}$ and William D Beavis ${ }^{* \dagger}$ \\ * Department of Agronomy \\ ${ }^{\dagger}$ Bioinformatics and Computational Biology Graduate Program}

Iowa State University, Ames, Iowa, USA - 50010

Factors affecting Response to Recurrent Genomic Selection in Soybeans

Keywords: Genomic Selection, Genetic Response, Recurrent Selection, Soybean, Nested Association Mapping

\section{Corresponding Author:}

Vishnu Ramasubramanian

1525, Agronomy Hall, ISU

Ames, IA 50011

Phone: 5155986376

Email: vishnur@iastate.edu 


\section{Abstract}

2 Herein we report the impacts of applying five selection methods across 40 cycles of recurrent

3 selection and identify interactions among factors that affect genetic responses in sets of

4 simulated families of recombinant inbred lines derived from 21 homozygous soybean lines. Our

5 use of recurrence equation to model response from recurrent selection allowed us to estimate the

6 half-lives, asymptotic limits to recurrent selection for purposes of assessing the rates of response

7 and future genetic potential of populations under selection. The simulated factors include

8 selection methods, training sets, and selection intensity that are under the control of the plant

9 breeder as well as genetic architecture and heritability. A factorial design to examine and analyze

10 the main and interaction effects of these factors showed that both the rates of genetic

11 improvement in the early cycles and limits to genetic improvement in the later cycles are

12 significantly affected by interactions among all factors. Some consistent trends are that genomic

13 selection methods provide greater initial rates of genetic improvement (per cycle) than

14 phenotypic selection, but phenotypic selection provides the greatest long term responses in these

15 closed genotypic systems. Model updating with training sets consisting of data from prior cycles

of selection significantly improved prediction accuracy and genetic response with three

17 parametric genomic prediction models. Ridge Regression, if updated with training sets consisting

18 of data from prior cycles, achieved better rates of response than BayesB and Bayes LASSO models. A Support Vector Machine method, with a radial basis kernel, had the worst estimated

20 prediction accuracies and the least long term genetic response. Application of genomic selection

21 in a closed breeding population of a self-pollinated crop such as soybean will need to consider

22 the impact of these factors on trade-offs between short term gains and conserving useful genetic

23 diversity in the context of the goals for the breeding program. 


\section{Introduction}

26 Genetic improvement in commercial and public soybean breeding programs include variety

27 development as the evaluation phase of recurrent selection (Orf 2008). Each cycle consists of

28 making crosses among selected lines, creating replicable homozygous lines by self-pollinating

29 progeny for several generations, evaluating the lines in replicated field trials for several years

30 during which poor performing lines are discarded and superior performing lines are retained to

31 cross and begin a new cycle. Pedigrees of modern soybean varieties confirm that genetic

32 improvement of soybeans in Maturity Zones (MZs) II, III and IV has been largely through intra-

33 population recurrent selection (Byrum, personal communication; Hyten et al. 2006; Mikel et al.

34 2010; Langewisch et al. 2017; Achard et al. 2020). Prior to the Plant Variety Protection Act in

351970 (https://www.ams.usda.gov/rules-regulations/pvpa), a cycle of genetic improvement would

36 require from 10 to 14 years, whereas in the last 40 years commercial organizations have invested

37 in development of continuous nurseries including software that streamlines inventories and

38 logistics of seed transfer, resulting in the capacity to complete a cycle of recurrent selection in

five years (Byrum et al. 2017; Anderson et al 2019). Thus, using current best practices, a

40 soybean breeder might experience three to five cycles of genetic improvement.

41 Despite reducing cycle times to less than $1 / 2$ of the time required 40 years ago, yield

42 improvements for soybean have not doubled in the corn-soybean agricultural system of the

43 United States (Mikel et al. 2010; Specht et al. 2014). Recognizing the relatively slow yield

44 improvements, farmer-members of the North Central Soybean Research Program (NCSRP)

45 provided funding to public soybean breeders to identify novel QTL in germplasm adapted to

46 MZ's II, III and IV and subsequently to identify methods to increase soybean yields

47 (https://www.ncsrp.com/NCSRP_research.html\#yield). The SoyNAM project provided evidence 
48 of novel useful genetic variability for yield among the SoyNAM lines (Song et al. 2017; Diers et

49 al. 2018) and genomic selection (GS) is being investigated for improving yield in pubic

50 germplasm.

51 After the seminal work by Bernardo and Yu (2007), crop breeders have experimentally

52 demonstrated the relative advantage of GS to phenotypic selection (PS) for a few cycles of

53 recurrent selection in barley, maize, oats, rice and wheat (Bernardo 2008, 2014; Asoro et al.

54 2011; Heslot et al. 2012, 2015; Nakaya and Isobe 2012; Emily and Bernardo 2013; Crossa et al.

55 2014; Beyene et al. 2015; Bassi et al. 2016; Marulanda et al. 2016; Jonas and de Koning 2013,

56 2016; Hickey et al. 2017; Goiffon et al. 2017). Genomic prediction (GP) has been integrated in

57 commercial soybean variety development projects as a tool to identify crosses that are most

58 likely to produce superior progeny and to a lesser extent as a means to reduce the costs of

59 conducting preliminary field trials (Kurek 2018). However, implementation of GS in soybeans

60 has not been based on an understanding of how various factors and their interactions can affect

61 responses to recurrent GS (RGS) in a crop with population structures and genome organizations

62 such as found in soybean.

63 Simulations have been used to demonstrate that genome organization, population structure,

64 genetic architecture, heritability, selection intensity, GP models and composition of training sets

65 will affect responses to selection (Robertson 1960; Dempfle 1974; Kang 1979; Cockerham \&

66 Burrows 1980; Kang and Namkoong 1980; Kang 1987; Kang and Nienstaedt 1987; Podlich and

67 Cooper 1998; Cooper et al. 2002; Habier et al. 2007; Goddard 2009; Zhong et al. 2009; Jannink

68 2010; Bastiaansen et al. 2012; Bijma 2012; de los Campos et al. 2013; Howard et al. 2014; Liu et 
responses across a few cycles of recurrent selection, although a couple of studies investigated

71 limits of responses to RGS across more than 20 cycles (Jannink 2010; Liu et al. 2015).

72 Comparisons of genomic prediction (GP) models have revealed that simulated genetic

73 architecture and proportion of phenotypic variance due to the genotypic variance affected

74 prediction accuracies and simulated responses to selection (de los Campos et al. 2010, 2013;

75 Wimmer et al. 2013; Howard et al. 2014). Parametric methods such as Ridge Regression (RR) and Bayesian regression in the mixed effects modeling framework provide more accurate

77 predictions and greater gains for traits with additive genetic architecture, whereas non-parametric machine-learning methods such as Neural Networks and Support Vector Machines provide more

79

80

81 accurate predictions for traits with epistatic genetic architectures (Howard et al. 2014). Prediction accuracies are essentially the same for all GP models applied to data with additive genetic architectures (Long et al. 2010, 2011; Guo et al. 2012; Howard et al. 2014). Given additive genetic architectures, RR (Endelman 2011) and Bayesian models (Pérez and de los Campos, 2014) provide similar short-term genetic gains, but different responses after $\sim 15$ cycles of RGS.

Marker densities impact prediction accuracies and short term genetic gains, with a dense marker set performing better than a sparse set, although there are limits to improvements from increased marker densities that depend on linkage disequilibrium (LD) and structure of the breeding population (de Roos et al. 2009; Schulz-Streeck et al. 2012; Hickey et al. 2014; Xavier et al, 2016; Norman et al. 2018). Decreased prediction accuracies of GP models in later cycles of recurrent GS are associated with decay of LD between marker loci (ML) and quantitative trait loci (QTL), loss of relationships between lines in early and later cycles of selection or a combination of both (Habier et al. 2007; Zhong et al. 2009; Hickey et al. 2014; Liu et al. 2015; Müller and Melchinger 2017, 2018). It is possible to offset the loss of relationships and LD, by 
93 updating the training sets with each cycle of selection (Jannink 2010; Liu et al. 2015; Müller et al. 2017, 2018). If training data are from only the current cycle of selection, then predictions do not take into account relationships between the current population and the founder population or possible loss of LD. At the other extreme, data from all prior cycles of selection can be included with data from the current cycle in the training set, but practical computation limits will be encountered with large training sets.

With the development of male sterile and insect pollination systems for soybean, it will become possible to conduct one to three cycles of RGS per year (Ortiz-Perez 2008; Davis, personal communication). This will enable increased selection intensities and greater immediate genetic gains from GS because much larger numbers of progeny can be evaluated without the commensurate added expense of increased numbers of field plots (Heslot et al. 2015). However, it is well-established that increased selection intensities will reduce the genetic potential of a breeding population through loss of useful genetic variability and ultimately will limit responses to selection (Robertson 1960; Hill and Robertson 1968; Bulmer 1970). useful genetic variance from selection, it is important to recognize that expression of novel 112 genetic modifications within genetic networks depend on the genomic background, even in 113 simple model organisms such as yeast (Peccoud et al. 2004; Forsberg et al. 2017; Hou et al. 114 2018). Since RGS with one to multiple cycles per year (Gaynor et al. 2017) will become feasible 115 for soybean and because genome editing will need genetic diversity for success, soybean 
breeders need to determine appropriate trade-offs between short term genetic gains and retention

117 of useful genetic variability in the germplasm that will be inherited by future plant breeders

118 (Rodríguez-Leal et al. 2017; Lemmon et al. 2018).

119 In anticipation of emerging technologies that will enable soybean breeders to implement a two

120 part strategy for genetic improvement (Gaynor et al. 2017), we investigated the impacts of

121 selection methods (PS and GS), training sets (TS), number of QTL, heritability (H), selection

122 intensity (SI) and their interactions on responses to RGS. We employed a factorial treatment

123 design, where each combination of factor levels was replicated ten times in simulated RGS and

124 RPS of recombinant inbred lines (RILs) across 40 cycles. Outcomes reported herein provide a

125 foundation for comparisons with strategies that use other types of progeny for selection and

126 crossing as well as practical guidelines for understanding trade-offs between genetic gains in

127 early cycles and retention of useful genetic potential for future genetic improvement of soybeans.

128 Methods

129 Simulations and Treatment Design. The impact of nQTL, SI, h, TS and SM on response to

130 selection across 40 cycles of recurrent selection were evaluated with a factorial design consisting

131 of 306 combinations of factors. In contrast to evaluating one or two variables at a time, the

132 factorial design is widely used to comprehensively determine factors that will optimize processes

133 (Myers 1976; Collins et al. 2014; Dunn 2020). Specifically the treatments consisted of three

134 values for number of simulated QTL, three selection intensities, two values for non-genetic

135 variance, five selection methods and four types of TS used to update four GP models (Table 1).

136 In summary the treatment design consists of 18 combinations of factors for PS plus 288

137 combinations of factors for GS methods for a total of 306 combinations of factors. Note that TS 
are irrelevant for PS. Each set of factor combinations was replicated with ten simulated recurrent selection projects across 40 cycles resulting in 3060 simulations with 122400 outcomes.

Simulated soybean RILs were generated by crossing in silico 20 homozygous SoyNAM founder lines with IA 3023 to generate 20 distinct $F_{1}$ progeny. The $F_{1}$ progeny from each of the 20 crosses were self-pollinated in silico for five generations to generate 100 RILs per family. The resulting 2000 RILS from 20 families were assessed for segregating genotypic information at 4289 SNP loci (Song et al. 2017; Xavier A et al. 2017; Diers B et al. 2018). On average alleles from the common founder occurred at a frequency of 0.9 and alleles at the loci from all other founder lines occurred at a frequency of 0.1. Soybeans has been subjected to phenotypic selection and genetic improvement for several thousand years (Anderson et al. 2019). Thus, the SoyNAM founders represent a realized population structure adapted to maturity zones II, III and IV that is akin to a single coalescent process used in many simulation studies (Daetwyler et al. 2013; Bandillo et al. 2017).

Of the 4289 SNP markers with genotypic scores for the SoyNAM population 3818 were polymorphic among the 20 families used as founders for the simulations. On average, 773 were polymorphic for a family with a standard deviation among families of 34 . In the initial founding set of RILs, the average heterozygosity per SNP locus across 20 families was 0.09 . 'Gst' is a measure of sub-population differentiation estimated as ratio of difference between expected heterozygosity of sub- populations to total expected heterozygosity. The average estimated $\mathrm{G}_{\text {st }}$ value across the genome for the initial founding set of RILs was 0.32 , as determined by the 'diff_stats' function in the mmod R package (Jombart 2008; Ryman and Leimar 2009; Jombart and Ahmed 2011). Relative to previous reported founders that used a coalescent process (Woolliams and Corbin 2012; Hickey and Gorjanc 2012; Daetwyler HD et al. 2013), our 
161 simulations began with a structure more likely to be found in actual soybean breeding

162 populations and with less, albeit more realistic, genetic diversity of soybeans adapted to maturity

163 zones II, III and IV. We've also estimated Pairwise 'Fst' using 'pairwise.fst' in 'hierfstat' R

164 package (Goudet 2005), which is a measure of population differentiation among pairs of

165 populations. It is estimated as the ratio of difference between the average of the expected

166 heterozygosity of the two populations and total expected heterozygosity of the pooled

167 populations to total expected heterozygosity of the pooled populations. Average Fst among the

16820 families in simulated SoyNAM data is 0.20 . Whereas the average Fst using genotypic data

169 from SoyNAM project among 40 families is 0.09 with a maximum pairwise Fst of 0.15 and a

170 minimum Fst of 0.007. Average Fst among the clusters in USDA soybean germplasm collection

171 is $0.22-0.23$ (Song et al 2015; Xavier 2018).

172 Subsets of 40, 400, and 4289 SNP loci were designated as QTL. The QTL were distributed

173 evenly throughout the genome, and each contributed equal additive effects of 5/-5, 0.5/-0.5, or

$174 \quad 0.05 /-0.05$ units respectively to the total genotypic value of the simulated RILs. Thus, all three

175 genetic architectures had the same potential to create genotypic values ranging from +200 to -

176200 in the initial founder sets of RILs. Positive and negative allelic effects were simulated to

177 alternate sequentially at QTL that are uniformly distributed across the Soybean genetic map.

178 Because all marker alleles are QTL alleles when there are 4289 QTL, LD between marker alleles

179 and QTL will not deteriorate across cycles of selection and recombination. Phenotypic values

180 were simulated by adding non-genetic variance sampled from an $\mathrm{N}(0, \sigma)$ distribution to the

181 simulated genotypic values, where $\sigma$ was determined by the heritability on an entry mean basis

182 among the initial sets of founder sets of RILs. Broad sense heritability on an entry mean basis

$183(\mathrm{H})$ values of 0.7 and 0.3 were simulated for each of the three sets of QTL. After the phenotypic 
184 values were simulated in the initial founding RILs, the non-genetic variance was held constant across subsequent cycles of selection.

186 For each cycle of recurrent selection, $1 \%, 2.5 \%$ or $10 \%$ of the most positive phenotypic or 187 predicted phenotypic values among 2000 simulated RILs, corresponding to selection intensities of 2.67, 2.34 and 1.75, were selected as lines to inter-mate for the next cycle. Matings among pairs of selected RILs used a design with greater contributions from the best selected lines (Figure 1) also known as networked families designs (Guo et al. 2013; Guo et al. 2014). models and Support Vector Machine with Radial Basis Kernel (SVM-RBF) represented a nonparametric method of machine learning.

Ridge regression was implemented with a method that employs expectation maximization to obtain Restricted Maximum Likelihood estimates of marker effects (Xavier et al. 2019). This computational method is faster than the popular implementation of ridge regression in rrBLUP package (Endelman 2011) and generates values that are highly correlated with the predictions based on the rrBLUP package (Figure S1). The BGLR package (Perez and de los Campos 2014)

201 provided implementations of BB and BL models. The 'Rgtsvm' package in R was used for its implementation of the SVM with RBF kernel method (Wang et al. 2017). 'Rgtsvm' implements SVM training on GPUs with computing time several hundred times less than that required for the

204 implementation in the 'caret' package on high performance computing clusters, and produces 205 similar prediction accuracies and estimates of mean squared errors (Figure S2). The parameters used to train GP models with R packages are provided in Table 2. 
207 For purposes of this manuscript we use the phrase 'model updating' to refer to retraining GP

208 models with up to 14 previous cycles of training data (Figure S3). A preliminary analysis of TS

209 on genotypic values and prediction accuracies was conducted using RR-REML models trained

210 with data from the current cycle as well as 3, 5, 8, 10, 12, and 14 prior cycles. The results were

211 compared with responses from the RR-REML model updated with TS's comprised of data from

212 all prior cycles and a RR-REML model with no updating. Training sets for each cycle were

213 obtained by randomly sampling 1600 RILs from the set of 2000 simulated RILs in each cycle.

214 The most accurate prediction and maximum genetic response was obtained with training data

215 that is cumulatively added every cycle (Figure S4 and S5). The results indicate that 3-5 prior

216 cycles of training data did not significantly improve prediction accuracies and responses relative

217 to models that were not updated. Also, the standardized genotypic values and prediction

218 accuracies, obtained using 10 to 14 prior cycles of data in the TS's, were not significantly

219 different than results based on TS's consisting of all prior cycles. Based on the results of this

220 preliminary study, we investigated responses to recurrent selection using TS's consisting of up to

22114 prior cycles of selection as well as data from the current cycle. After the $14^{\text {th }}$ cycle, training

222 data consisted of 14 prior cycles of recurrent selection.

223 Modeled response to recurrent selection. The averaged genotypic value for each cycle, c, of

224 recurrent selection was modeled with a linear first order recurrence equation:

$$
f_{0}(\mathrm{c}) y_{(\mathrm{c}+1)}+f_{1}(\mathrm{c}) y_{(\mathrm{c})}=g(\mathrm{c}) \quad(\text { Eqn } 1)
$$

226 Where $\mathrm{c}$ is a sequence of integers from 0 to 39 representing each cycle of recurrent selection

227 from cycle 1 to 40 and $f_{0}, f_{1}$ and $g$ are constant functions of c. By rearranging the equation we

228 note that the response in cycle $\mathrm{c}+1$ can be represented as 


$$
y_{(\mathrm{c}+1)}=-\frac{f_{1}(\mathrm{c})}{f_{0}(\mathrm{c})} y_{(\mathrm{c})}+\frac{g(\mathrm{c})}{f_{0}(\mathrm{c})}
$$

230 Since the ratios $f_{1}(\mathrm{c}) / f_{0}(\mathrm{c})$ and $g(\mathrm{c}) / f_{0}(\mathrm{c})$ are constants, we can represent the response in cycle $\mathrm{c}+1$

231 as

$$
y_{(\mathrm{c}+1)}=\alpha y_{(\mathrm{c})}+\beta
$$

$$
\begin{aligned}
& y_{c}=\alpha^{c} y_{0}+\beta \frac{1-\alpha^{c}}{1-\alpha} \text { if } \alpha \neq 1 \\
& y_{c}=\alpha^{c} y_{0}+\beta c \quad \text { if } \alpha=1
\end{aligned}
$$

236 An alternative representation of (4) for the situation of $\alpha \neq 1$ is

$$
\begin{aligned}
& y_{\mathrm{c}}=\alpha^{\mathrm{c}}\left(y_{0}-y^{\prime}\right)+y^{\prime} \\
& \text { with } y^{\prime}=\frac{\beta}{1-\alpha},
\end{aligned}
$$

238 , where $\alpha$ is less than 1 for genotypic response to recurrent selection and $y^{\prime}$ represents the 239 asymptotic limit to selection (Goldberg 1958). To illustrate, values of the sequence of c=0 to 39 , 240 with $y_{0}=0$, for the range of $\alpha(0.6-0.9)$ and $\beta(1.4-38)$ values are plotted in Figure 2 . The curves

241 can be interpreted as response to selection as a function of the frequencies of alleles with additive 242 selective advantage, selection intensity, time and effective population size (Robertson 1960).

243 The parameters, $y_{\mathrm{o}}, \alpha$, and $\beta$, were estimated with a non-linear mixed effects method

244 implemented in 'nlme' functions in the 'nlme' and 'nlshelper' packages (Pinheiro and Bates

245 2000; Baty et al. 2015; Pinheiro et al. 2019). 
246 Since the limits of responses are asymptotic, the number of cycles before there is no longer

247 response to selection is referred to as the half-life (Robertson 1960; Dempfle 1974; Kang 1979;

248 Cockerham \& Burrows 1980; Kang and Namkoong 1980; Kang 1987; Kang and Nienstaedt

249 1987). From the first order recurrence equation, the half-life is estimated as $\ln (0.5) / \ln (\alpha)$, when

$250 \mathrm{y} 0$ is ' 0 ' and the asymptotic limit is estimated as $\mathrm{y}$ '.

251 Analyses of variance (ANOVA) of modeled response to recurrent selection. The purpose of the

252 ANOVA is to evaluate the impact of factors and their interactions on the modeled responses to

253 recurrent PS and GS methods. The analyses of variance used single and multi-level nlme models

254 with modeled (eqn 4) responses grouped by treatment factors. The influence of multiple factor

255 treatment combinations on estimated non-linear mixed effect models have not been implemented

256 in standard statistical software packages that report the analysis of variance in terms of sums of

257 squares and traditional 'F-tests'. For discussions on the challenges of using standard F-test for

258 non-linear mixed effects (nlme) models see (Pinheiro et al. 2000; Baty et al. 2015; Pinheiro et al.

259 2019). Consequently, we analyzed the variance among modeled responses using AIC, BIC and

260 Likelihood metrics that were grouped based on combinations of factors consisting of selection

261 methods, TS, SI, nQTL and simulated $\mathrm{H}$.

262 In order to provide a balanced data table for analyses by the non-linear mixed effect model,

263 responses that included PS, which has no TS's, were assumed constant resulting in a balanced

264 full factorial set of responses for 360 combinations of factors. The process of fitting, selecting

265 and refining mixed effects models closely followed the steps described in Pinheiro et al. 2000;

266 Zuur 2009 and Oddi et al. 2019). The complete process used in the study is provided in File S1.

267 In the first phase of model fitting, estimates of modeled parameters from nlsList models were

268 retained as starting values for fixed effects. Both alpha and beta were fit only for intercept and 
269 deviations from estimated means conditioned on grouping variables were modeled as random

270 effects using the 'nlme' R package. Multiple ANOVA of 'nlme' objects representing the models

271 were used to identify combinations of factors with significant effects on the non-linear response

272 model. The model with the lowest AIC score was selected as the best model. The best random

273 intercept model in the first phase of model fitting process M31 in Table S1 was further refined

274 by modelling the correlation structure.

\section{Evaluation of Simulated Response to Recurrent Selection}

276 While the modeled genotypic values are evaluated using half-life and asymptotic limits, we have

277 evaluated the simulated outcomes from recurrent selection using a set of metrics to assess

278 responses, population characteristics, and GP model performance every cycle of selection.

279 The standardized genotypic value, $\mathrm{R}_{\mathrm{s}}$ (eqn 5), was estimated every cycle as the change in

280 genotypic value from the average genotypic value of 2000 RILs derived from the initial founders

281 and standardized to the maximum genotypic potential (200 units) among the founders

282 (Meuwissen et al. 2001; Liu et al. 2015).

$$
\mathrm{R}_{\mathrm{s}}=\frac{\mathrm{R}_{\mathrm{c}}}{\left(\mathrm{R}_{\mathrm{m}}-\mathrm{R}_{0}\right)}
$$

$\mathrm{R}_{\mathrm{S}}$ - Standardized genotypic value

The most positive genotypic value $\left(M_{g v}\right)$ among RIL's selected in cycle c is a metric used to 
289 initial population, was used to evaluate the loss of genotypic variance. Note that values for the

$290 S V_{g}$ range from zero to one.

291 Response standardized to change in standard deviation of genotypic values captures genetic gain

292 with respect to loss of genetic variance (eqn 6). The numerator term represents change in

293 genotypic values of a population in cycle ' $c$ ' from cycle ' 0 ' founder population normalized to

294 standard deviation of genotypic values in cycle ' 0 '. The denominator term represents change in

295 standard deviation of genotypic values from cycle '0' to cycle 'c' as a fraction of standard

296 deviation of genotypic values in cycle 0 . This metric is similar to the metric used to refer to

297 efficiency of converting loss of genetic diversity to genetic gain of a selection method in

298 recurrent selection (Gorjanc et al. 2018). While efficiency is estimated as slope in linear

299 regression model with numerator as ' $y$ ' term and denominator as ' $x$ ' term in the linear part of

300 response curve, with Rs_Var it is possible to visualize both linear and non-linear sections of the

301 response curve.

302

$$
\text { Rs_var }=\frac{\mathrm{G}_{\mathrm{c}}-\mathrm{G}_{0}}{\mathrm{SdG}_{0}-\mathrm{SdG}_{\mathrm{c}}}
$$

$\mathrm{G}_{\mathrm{c}}$-average genotypic value of the set of RILs evaluated in cycle $c$ 
Estimated Linkage disequilibrium (LD) among pairs of marker loci on all 20 chromosomes was evaluated as the deviation of observed gametic frequency of alleles at a pair of loci from the product of the individual allele frequencies, assuming independence (Weir 1996). The R function 'get_PG_LD_StatsGSMethods' used to estimate pairwise LD between markers is provided in the R package 'SoyNAMPredictionMethods'. GP models were assessed using the estimated prediction accuracies $\left(\mathrm{r}_{p s}\right)$, defined as the estimated linear correlation (Pearson) between

311 predicted and simulated phenotypic values and the estimated Mean Squared Error (MSE), and

312 defined as the mean of the squared deviations of the predicted phenotypic values from the 313 simulated values.

\section{Analyses and Data Availability}

315 More information on the analyses can be found in the R package 'SoyNAMPredictionMethods'.

316 Also, simulated data and code are available as part of the package (File S1 found at

317 http://gfspopgen.agron.iastate.edu/SoyNAMPredictionMethods_v2_2020.html). Supplemental material including the R package can be found at https://figshare.com/s/f7d4e515e563beef550b and http://gfspopgen.agron.iastate.edu/SoyNAMPredictionMethods_v2_2020.html describes

320 how to use the package. The complete process for fitting NLME models and ANOVA can be 321 found at http://gfspopgen.agron.iastate.edu/Vishnu_MS1/NLME_Models_Part_I.html .SoyNAM 322 genotypic and phenotypic data are available in SoyBase (Grant et al. 2010).

\section{Results}

Prediction accuracies in the founding sets of RILs: Estimates of prediction accuracies, $\mathrm{r}_{\mathrm{ps}}$, of GP 
with BB and poorest with the SVM-RBF. The nQTL had little effect on $r_{p s}$ within either value of GP models are trained without QTL in the training set, but follow a similar pattern as the models with QTL. MSE are greater or comparable for models trained without QTL than for models with QTL (Figure S6- S7). Average within family prediction accuracies are lesser than prediction accuracies from a combined TS comprising of RILs from all the families (Figure S8-S9). However, a combined TS will have ( $n$ *population size of family) for ' $n$ ' families and estimated accuracies will have confounding effects from training set size. Estimated accuracies for models trained with TS generated by random sampling from a combined population to keep the TS size

337 same as family size are lower than average within family accuracies. MSE are lesser for combined TS than for models trained using within family TS and sampled TS (Figure S8-S9).

Influence of factors on response metrics. The averaged responses Rs were modeled (eqn 4) and increase of Rs across the first five cycles of selection followed by slower responses from cycles 5 to 10 and no response after cycle 20 .

343 While there are observable general trends for each of the individual factors, response metrics are 344 unique for each combination of all factors (Figure 4-7, S10 - S14). The most parsimonious

345 model requires unique estimates of $\alpha$, and $\beta$ (eqn 4 ) for each of the combinations of factors

346 indicating that interactions among all factors have significant influences on the responses (Table 347 S1). Also analyses of variances on subsets of 10 and 20 cycles of selection demonstrate that the 
importance of factors on interaction effects were consistent (nQTL $>$ SM $>$ SI $>H>T S$ ) and

Among the three factors SM, TS and SI that are under the control of the plant breeder, we

Relative to PS, the three parametric GP models provided greater initial rates of response, reduced consistently produced the least effective responses (Figure 4-7). Training sets consisting of data cycle of selection are observably distinctive for both the short term and long term response metrics (Figure 4-7). Importantly, use of the TS's significantly improved responses to selection

362 in both the short term and long term for all other combinations of factors (Table S1). Selection 363 intensity is the third most important factor to affect the response metrics (Table S1). Consistent 364 with theory (Robertson 1960; Hill and Robertson 1968; Bulmer 1971,1976), greater SI's

365 (associated with retention of smaller numbers of RILs to initiate subsequent cycles, were 366 associated with more rapid response rates, shorter half-lives, faster loss of genetic variance and 367 significantly lower Rs values as the population approached it's limit to respond to selection. greatest deviations from the average response (Table S1). From the modeled responses the half- 
3714289 QTL it ranged from 2.2 to 8.8 and 3 to 9.5 cycles respectively. The modeled responses

372 were highly correlated with the simulated responses (Pearson correlation coefficient: 0.94-0.97).

373 To illustrate the impact of nQTL, consider Rs values plotted across forty cycles of recurrent

374 selection (Figure 4 and S15). If the genetic architecture of the trait consists of 40 and 400 QTL,

375 responses to selection were limited after 10-15 cycles of selection, whereas for 4289 QTL, limits

376 to selection responses were not realized until 30 to 40 cycles of selection.

377 Next consider that the maximum realized response for 40 simulated QTL was from 0.32 to 0.78

378 of maximum genotypic potential among the founders, whereas for 400 and 4289 QTL, the

379 realized response was $0.12-0.42$ and $0.04-0.17$ of the maximum (Figure 4 and S15). Also if

380 there are 40 simulated QTL, the maximum attained values are as high as $80 \%$ of the maximum

381 value of 200 in less than ten cycles of recurrent selection (Figure 8 and S16). In contrast, Rs

382 values are no greater than $40 \%$ of the maximum value and stop responding to selection in 10-15

383 cycles if there are 400 simulated QTL. Rs values were never greater than $15 \%$ of the maximum

384 value and only begin to approach a limit after 20 cycles if there are 4289 simulated QTL.

385 As expected, responses to selection reflect declining genetic variances (Figure 9 and S17). The

386 loss of Sgv's across cycles is much faster with fewer simulated QTL than larger numbers of

387 QTL. Likewise the estimated prediction accuracies (Figure 10 and S18) approach zero as the

388 genotypic variance approaches zero. Average MSE for the GP models increase across cycles of

389 selection (Figure 11 and S19) LD among markers approach zero as the genotypic variance

390 approaches zero although the covariance among these response metrics depend on the other

391 simulated factors (Figure S20-S24). 
392 To interpret the role of nQTL as a factor, it is important to recall that: 1) positive and negative

393 allelic effects were simulated to alternate sequentially at QTL (marker loci) that were distributed

394 across the genome according to the Soybean genetic linkage map. 2) Crossing nearly

395 homozygous lines and subsequently self-pollinating progeny for five generation before

396 genotyping and phenotyping within each cycle creates a limited number of large linkage blocks.

397 Analyses of the number of linkage blocks each generation reveals that regardless of the number

398 of QTL, the number of linkage blocks per cycle ranges from 70-90. It was not the same blocks

399 each cycle, but if the nQTL equals 40, then each linkage block included all segregating QTL

400 each cycle. For 400 and 4289 simulated QTL, each linkage block had a net genetic effect of zero

401 or +/- 0.5 or +/- 0.05 multiplied by the number of QTL in the block respectively. Thus, the nQTL

402 might be better understood as the magnitude of genetic effects associated with segregating

403 linkage blocks.

404 The observable general trend for $\mathrm{H}$, or perhaps more accurately understood as contributions of

405 non-genetic effects to the phenotypes, was that $\mathrm{H}$ values of 0.7 in the initial phenotypic variance

406 resulted in Rs values that were greater than $H$ values of 0.3 of the initial phenotypic variance

407 (Figure 4 and S15). The trend in Rs values is correlated with the other response metrics, in

408 particular prediction accuracies of the GP models. The loss of estimated prediction accuracies

409 are greater with $\mathrm{H}$ values of 0.3 than 0.7 with relaxed selection intensities (Figure 10 and $\mathrm{S} 18$ ).

410 Other combinations of SI and H require model updating to provide reasonable GP model

411 prediction accuracies and achieve greater responses across more cycles of selection. As we

412 would expect, for all combinations of SI and nQTL, loss of genotypic variance are greater with $\mathrm{H}$

413 values of 0.7 than 0.3 (Figure 9 and S17). 
414 Some specific outcomes of interest: For purposes of illustrating interaction effects on SM's

415 across all 40 cycles of selection, consider the most relaxed SI of 1.75, associated with selecting

$41610 \%$ of the RILs per cycle. When GP models are not updated, BB produced greater $\mathrm{R}_{\mathrm{s}}$ values

417 than PS in the early cycles for all nQTL and both levels of H, whereas PS resulted in greater

418 responses than all GS methods after the $10^{\text {th }}$ cycle (Figure 6, S15 and S25). SVMRBF did not

419 demonstrate any better responses than PS in either early or late cycles for any nQTL or level of

$420 \mathrm{H}$ (Figure 6, S15, and S25; File S3 and S4).

421 If the parametric GP models are updated with training sets consisting of data from up to 14 prior

422 cycles of recurrent selection, responses to RR-REML demonstrated the greatest responses (Rs)

423 for 40, 400 and 4289 QTL across both levels of $\mathrm{H}$ (Figure 7, S15 and S26; File S3 and S4). If

424 the RR-REML model is updated with up to 14 prior cycles of training sets, responses are larger

425 than PS for up to 10-40 cycles depending on the number of QTL, H and SI (Figure 4 and 7; File

426 S3 and S4). When BB and BL GP models are updated, responses are larger than PS for up to 5,

42720 and 40 cycles for 40, 400, and 4289 QTL respectively. Similar, albeit distinctive, comparisons

428 among outcomes from GP models with model updating for genetic architectures responsible for

4290.3 of the phenotypic variance in the initial sets of RILs (Figure S26) are described in File S4.

430 Relative to responses without model updating application of RR-REML and Bayesian methods

431 with model updating resulted in greater responses. Model updating with Bayesian methods also

432 resulted in less favorable responses than the RR-REML (Figure S27 and S28; File S4).

433 SVMRBF when updated with TS's demonstrated no significant improvement relative to

434 SVMRBF without updating for all genetic architectures, levels of H and SI's (Figure S27 and

435 S28; File S4). If the genetic architecture explains only $30 \%$ of the phenotypic variability in the

436 initial sets of RILs, the relative improvements in Rs values across cycles using updated TS's are 
437 better than simulated QTL that explain $70 \%$ of the phenotypic variance (Figure S28). Percentage

438 gain in responses for GS with model updating relative to response from GS without model

$439 \quad$ updating are provided in File S5.

440 In terms of lost genetic potential, every cycle of selection reduced the maximum possible genetic

441 value. When GP models are not updated, the genetic potential is lost at a rapid rate beginning in

442 the early cycles, whereas when GP models are updated, genetic potential is retained in the

443 population and genetic variance decreases at a slower rate. PS had the least loss in genetic

444 potential relative to all four GP models without updating. However, with model updating, the

445 loss of genetic potential using parametric GP models was almost the same as PS. Among the

446 parametric GP models, RR-REML and Bayesian methods showed similar slow losses of genetic

447 potential with and without model updating. SVMRBF GS had the greatest loss of genetic

448 potential beginning with the early cycles (Figure 5).

449 The loss of genetic potential in early cycles determines the limits to selection response in later

450 cycles. For example, with 400 simulated QTL responsible for $70 \%$ of the phenotypic variance,

451 the maximum potential was only $50 \%$ of the maximum potential (100 units) for PS and

452 parametric GS methods with and without model updating. When parametric GP models are

453 updated, $81 \%$ and $75 \%$ of the limits of maximum available potential are realized with RR-REML

454 and Bayesian methods respectively. If GP models are not updated, only $62 \%$ of the limits of the

455 maximum available potential are realized with RR-REML and Bayesian methods. With PS, 78\%

456 of maximum available potential is realized by the cycles in which the population no longer

457 responds to selection. With SVMRBF, only $35 \%$ of the potential is realized with and without

458 updating by the cycles in which the population no longer responds to selection (Figure 5). 
If GP models are not updated with data from up to 14 prior cycles, the Mgv's were consistently greater with PS than the four GS methods. Among GP models without updating, BB provided the best Mgv, while SVM-RBF had the smallest Mgv (Figure 8 and S16). If GP models are updated, the pattern depends mostly on the number of QTL. For initial H values of either 0.7 and 0.3 and 40 simulated QTL, Mgv's are similar for RR-REML, Bayesian GP models and PS,

464 whereas for 400 QTL, RR-REML produces greater Mgv's than PS and Bayesian methods. For 4289 QTL, RR-REML and Bayesian methods produce greater Mgv's than PS. Recurrent GS with SVMRBF produced the least desirable Mgv's for 40, 400 and 4289 QTL.

467 If GP models are updated, the standardized genotypic variance (Sgv) decreases at a rate similar 468 to GP models that are not updated (Figure 9 and S17). There is no difference among GS methods 469 in terms of rate at which Sgv decreases. Also, model updating significantly improved estimated 470 prediction accuracies, $\mathrm{r}_{\mathrm{ps}}$, for all GP models except SVMRBF. Among RR-REML and Bayesian

471 GP models, model updating has a slightly larger impact on estimated accuracies and MSE using

472 RR-REML than with Bayesian GP models (Figure 10, 11, S18 and S19). MSE were orders of

473 magnitude lesser for RR-REML than bayesian GP models with updates after the first 10-15

474 cycles of selection (Figure 11 and S19).

475 If models are updated using data from up to 14 prior cycles, the changes to genetic variance 476 among the RILs selected to be crossed, their average heterozygosity, average rate of inbreeding, 477 and loss of favorable alleles are similar among GS methods (Figure S29- S32). Model updating 478 resulted in faster loss of genotypic variance among genotypes selected to be parental lines for the 479 next cycle of inter-mating. The loss of genotypic variance is similar among parametric GS 480 methods. (Figure S29). When there are 400 simulated QTL responsible for $70 \%$ of the 481 phenotypic variance, the average number of favorable alleles that are lost across 40 cycles due to 
482 selection and drift are similar among PS and GS methods, but the rate at which they are lost

483 differs among selection methods for the first 20 cycles until they converge at the same limit.

484 SVMRBF GS showed the greatest rate of loss and PS had the least rate of loss while the

485 parametric GS methods had intermediate rates of loss. By the time 40 cycles of selection have

486 completed, model updating didn't result in any significant difference in rates of loss and the total

487 number of favorable alleles that are lost (Figure S30).

488

489

490

491

492

493

494

495

496

497

498

499

500

501

502

503

504

SVMRBF GS showed the greatest loss of average heterozygosity and PS lost heterozygosity at the slowest rate, while RR-REML and bayesian GS methods lost heterozygosity at intermediate rates. Model updating didn't result in significant changes to rates at which heterozygosity was lost over cycles (Figure S31). PS showed slower rates of inbreeding than GS methods as we would expect from the decay of standardized genotypic variance. Average rates of inbreeding were similar among parametric GP models in the early cycles of recurrent selection, whereas the patterns varied after there is no response to selection (Figure S32).

For all selection methods pairwise LD among markers on the same chromosome decreased across cycles of recurrent selection (Figure S20-S24). LD decreased slowest with PS (Figure S20). Loss of LD in early and late cycles of selection are similar among parametric GP models and SVMRBF with the relaxed selection intensity. By the $20^{\text {th }}$ cycle of recurrent selection, LD approached zero for all selection methods and there was no evidence that selection methods affected linkage disequilibrium (LD) differentially in the earlier cycles. The rates at which LD decays are lower when GP models are updated with training sets compared to GP models without updating (Figure S20-S24).

The limiting values for RsVar (Response standardized to change in genotypic variance) when PS is used to select the best $10 \%$ of RILS with genetic architectures consisting of 400 and 4289 
QTL are greater than the limiting values using GS methods without model updates (Figure S33

GS methods and are dependent on nQTL, SI and H (Figure S35 and S36). With 0.7 heritability,

512 there are no significant difference in RsVar among GS methods for 40 simulated QTL. If the

513 genetic architecture consists of 400 and 4289 QTL and weaker selection intensities are practiced,

514 the RR-REML GS method maintained genetic variance and RsVar for more cycles than PS and 515 the Bayesian GS methods. Relative Gain in RsVar with RR-REML GS is even larger for 0.3 H

516 treatment with relaxed selection intensities (Figure S35 and S36). SVMRBF demonstrated the

517 least limits of RsVar for treatment combinations with and without model updating (Figure S33 -

518 S36). The plots for all the evaluation metrics discussed above for selection intensities 2.67 and

5192.34 are provided in Figure S37 -S60 and discussed in File S6).

\section{Discussion}

523 Previous publications of in silico investigations of factors affecting outcomes from RGS have

524 been conducted using a few factors applied to arbitrary diploid genomes, and an expected

525 population structure from an assumed coalescent process. To our knowledge, the research

526 reported herein is the first designed to reveal interactions among five factors that previously had

527 been shown to affect responses to selection. Our ability to detect and characterize interaction 
528 effects is enabled by use of a first order recurrence equation (eqn 4). Hopefully, our explanation

529 of how to implement recurrence equation models in available R packages will encourage others

530 to investigate recurrent genetic improvement designs. Even though our motivation for the use of

531 non-linear modeling in this study was restricted to the systematic investigation of significance of

532 variation in response due to the factors and the relative order of magnitude of interaction effects

533 of factors, non-linear mixed effects models have predictive power that is still relatively

534 unexploited in the study of limits due to recurrent selection. We plan to investigate the use of

535 non-linear recurrence modelling in mixed model framework to estimate the magnitude of

536 interaction effects and optimize the use of genomic information at prediction and selection levels

537 to optimize response in recurrent selection schemes in the future.

538 Most in silico investigations of GS have simulated diploid genomes consisting of ten or fewer

539 linkage groups with evenly spaced molecular markers depending on the organism (Jannink 2010;

540 Liu et al. 2015; Akdemir and Sánchez 2016; Yabe et al. 2016). The organization of the soybean

541 genome is the result of ancient duplication events (Shoemaker et al. 1996; Grant et al. 2000;

542 Cannon and Shoemaker 2012) and consists of many copies of functional genes that are

543 distributed unevenly with high concentrations near the telomeric ends of 20 diploid

544 chromosomes. Also, most prior simulation studies created population structures using an

545 assumed coalescent process (Woolliams and Corbin 2012; Hickey and Gorjanc 2012; Daetwyler

546 et al. 2013). We used a realized structure representing adapted and selected lines by sampling the

547 SoyNAM founders.

548 Structures of plant populations are highly dependent on the reproductive biology. Indeed, plant

549 breeders design genetic improvement projects based on reproductive biology. Similar to many

550 cereal and pulse crops, the reproductive biology of soybean is primarily through self-pollination 
551 (Wilcox et al. 1979; Fehr 1980, 1991). The frequency of natural cross pollination in soybean is

552 only about 0.025 (Garber et al. 1925; Caviness 1966; Carlson and Lersten 1987; Ahrent and

553 Caviness 1994). Because crossing soybean lines is labor intensive and expensive

554 (https://www.youtube.com/watch?v=VnjGijF4KQI ) soybean breeder's use mating designs in

555 which only one or two elite varieties are crossed with a few dozen recently selected RIL's (Guo

556 et al. 2013; Guo et al. 2014). We refer to this as a hub network design. Soybean breeders

557 subsequently take advantage of natural self-pollination to create RILs with sufficient seed for

558 replicated evaluations across many environments.

559 Our simulations attempted to emulate cycles of selection, crossing, and self-pollination currently

560 conducted by commercial and academic soybean breeders. Relative to outcrossing species

561 selecting homozygotes to participate in a hub network within each cycle will have smaller

562 effective populations and retain LD for more cycles of recurrent selection. Future studies are

563 needed to determine if the significant interaction effects that we found are due to population

564 structure and genome organization.

Implications for application of GS for genetic improvement with current soybean breeding.

566 There has been considerable concern expressed about the limited genetic variability among

567 soybean genotypes adapted to specific maturity zones and recurrently selected over 75 years in

568 North America (Carter et al. 2004; Hyten et al. 2006; Mikel et al. 2010; McCouch et al. 2013).

569 The SoyNAM founders represent a sample of current improved breeding germplasm adapted to

570 and used for agricultural production in maturity zones II - IV (Song et al. 2017; Diers et al.

571 2018).

572 Despite concerns about limited genetic variability, our assessment of the half-lives of

573 populations suggest that even if only 40 QTL remain in the founders of the SoyNAM population, 
574 there is still genetic potential for response to selection for at least five cycles. If there are larger

575 numbers of QTL with smaller additive effects distributed among linkage blocks of SoyNAM

576 founders, then we can expect reasonable half-lives to RGS for 10 cycles (File S2)- Because

577 current soybean breeding methods require four to five years before RIL's are selected to

578 participate in crossing nurseries, soybean breeders can expect reasonable responses to selection

579 using samples from the SoyNAM population for 20 to 60 years. It is possible to obtain

580 reasonable estimates of half-lives and asymptotic limits using the parameters in eqn 4 with

581 sufficient number of simulated cycles of selection. The estimated half-lives and asymptotic limits

582 can be used to assess the impact of selection on genetic potential of populations in the long term.

583 In our simulations, the estimates of half-lives using 10 or 20 simulated cycles, are significantly

584 less accurate than estimates obtained with 40 simulated cycles of recurrent selection (Table S2).

585 Among the five factors we investigated, a soybean breeder can choose SM's, SI's and TS's.

586 Currently, plant breeders have little control on nQTL and H, because these parameters are

587 determined by the sample of germplasm and environments. While it is possible to adjust the

588 value of $\mathrm{H}$ on an entry mean basis by increasing/decreasing the number of replicates (Fehr 1991),

589 estimating the number of segregating QTL and the magnitude of their effects is difficult and

590 usually extremely expensive (Beavis 1994; Goring et al. 2001; Xu 2003). For a fixed budget, the

591 breeder will be faced with a trade-off between numbers of replicates and numbers of RIL's that

592 can be evaluated. In other words, $\mathrm{H}$ on an entry mean basis can be estimated, but not adjusted

593 without adding field plot resources, and nQTL and their contributions to genetic variance can be

594 estimated, but the estimates will be biased.

595 Although modulated by nQTL and H, PS consistently retained useful genetic variability across

596 many cycles of genetic improvement, Bayesian methods provided the fastest genetic gains in the 
short term and RR-REML provided a compromise between PS and Bayesian methods. SVM-

598

599

600

601

602

603

604

605

606

607

608

609

610

611

612

613

614

615

616

617

618

619

RBF should not be considered for genetic improvement for the additive genetic architectures in soybean population structures. Previously others have reported that long-term response using GS methods will be more limited than PS in closed populations (Goddard 2009; Zhong et al. 2009; Jannink 2010). We also observed that GS without updated TS's result in rapid loss of genetic variance in the initial cycles, which results in lower Rs values as responses to selection approach an asymptotic limit. When models were updated with TS's composed of data from prior cycles of selection, loss of prediction accuracy slowed for all values of nQTL and H (Figure 10).

Given similar rates of decreasing genetic variance among parametric GS methods, the different limits to selection response is possibly due to greater efficiency of retaining genetic diversity with RR-REML for later cycles, although for many combinations of factors the limits of response with RR-REML and Bayesian methods are about the same. Unlike previous studies, we noted in the initial cycles of RGS genetic gains and estimates of accuracy were similar using BL and RR-REML, whereas after 15 cycles genetic responses were not as limited with RRREML, probably because additive genetic variance was retained for later cycles of selection (Liu et al. 2015).

Replicated responses to high values of SI quickly reach a limit in five to ten cycles of recurrent selection. Replicated responses to lower values of selection intensity consistently result in greater gains over more cycles, indicating that genetic drift is the most likely mechanism for loss of genotypic variance. These constraints on plant breeding programs are well characterized (Brisbane and Gibson 1995; Hayes et al. 2009; Jannink 2010; Hung et al. 2012; Liu et al. 2015; Akdemir and Sánchez 2016; Yabe et al. 2016). 
620

621

622

623

624

625

626

627

628

629

630

631

632

633

634

635

636

637

638

639

640

641

642

Model updating with TS's from prior cycles improves the relationship between training sets and validation sets and thus improves responses to GS without updated training sets. While model updating doesn't significantly change the estimated half-life, model updating did produce greater responses standardized to the rate at which genotypic variance is lost in selected populations with updated RR-REML GP models (File S2; Table S3).

The choice of which combinations of SM, SI and TS depend on the objectives of the breeding program. If the objective is to enter and capture market share in a short time, then maintenance of genetic diversity is not important. Averaged over nQTL and $\mathrm{H}$, the greatest changes in Rs values, i.e., rate of genetic gain, were attained in early cycles using BayesB and SI $=2.84$, without model updating. Thus, if a soybean breeding project wants to maximize the rate of genetic gain for a single quantitative trait in a population derived from a sample of the SoyNAM founders, then application of the BayesB GS method and large SI is the best combination to meet the breeding objective. If the breeding project has a long term breeding objective to improve germplasm while maintaining useful genetic diversity for purposes such as providing useful germplasm for future generations or evaluating genome editing, then PS or GS with RR-REML with relaxed SI will be the best combination to meet the breeding objective. The greatest values for Rs were attained using the RR-REML model with model updating and SI $=1.75$. Last if the breeding project has multiple objectives for both immediate and longer term goals, then pareto optima among tradeoffs involving responses/cycle and retention of useful genetic variance for multiple traits need to be identified (Akdemir et al. 2019).

\section{Lessons for future simulation studies. In our simulations we assigned the same amount of time} to develop and evaluate RILs for GS as for PS. Application of PS usually requires field trials for three years before lines are selected for use in a crossing nursery. In practice, one of the 
643 advantages of GS relative to PS is that only subsets of RILs need to be phenotyped. Commercial

644 soybean breeding projects have used genotypic values obtained from GP models to cull lines in

645 as they are being self-pollinated and prior to the first stage of field trials (Kurek 2018). Also it is

646 possible to train or update GP models with lines derived in earlier filial generations thereby

647 requiring less time per cycle (Bassi et al. 2016). Even if both GS and PS require the same amount

648 of time to develop lines before phenotypic evaluation, GS methods can be conducted as soon as

649 phenotypic information is available from first year field trials (Heffner et al. 2009), while PS

650 usually requires phenotypic evaluations for three years before lines are selected for crossing

651 nurseries to begin a new cycle. Herein we did not investigate the trade-offs between less

652 accurate predictions from models trained with less extensive phenotyping or phenotyping with

653 lines derived in earlier filial generations. These practical adjustments from application of GS

654 methods to current soybean genetic improvement projects still need to be investigated.

655 Accuracies of GP models in the founding set of RILs were similar to that reported in previous

656 studies (Long et al. 2010, 2011; Guo et al. 2012; Howard et al. 2014). However, our estimates of

657 accuracies are larger as we've included QTL in our training sets. Relationship among selected

658 RILs and LD between marker loci (ML) and QTL are considered the two most important sources

659 of prediction accuracy. In previous studies, relationship among RILs had a greater effect on

660 prediction accuracies for RR-REML than BL, whereas accuracy of BL was more dependent on

661 LD and both components showed similar contribution to the accuracy of BayesB GP models

662 (Habier et al. 2007; Zhong et al. 2009; Liu et al. 2015). Similar to our results without model

663 updating, Bayesian GS methods resulted in greater responses as the populations approached

664 asymptotic limits (Meuwissen 1997; Li et al. 2008; Akdemir and Sánchez 2016). However, GP

665 model updating reduced the difference in rate of decrease of prediction accuracies among RR- 
REML and Bayesian GP models but there was no consistent pattern in relationship among selected RILs and rate of loss for LD to explain the observed estimates of prediction accuracies. However, GP model updating consistently resulted in lesser MSE for RR-REML than BB and BL GP models across all levels of SI, nQTL and H. This pattern is consistent with greater efficiency of converting loss of variance into gain with updated RR-REML GS method. In order to estimate the contribution of LD and linkage blocks to prediction accuracy of GP models will require a design similar to that employed by Müller et al. $(2017,2018)$ for synthetic populations. Populations with unrelated training and prediction sets with LD and SNP based relationship estimates showed low prediction accuracy and low genetic response in recurrent GS similar to GS without updating in this study. Whereas populations with relationship between training and prediction sets with LD and SNP based relationship estimates showed greater prediction accuracy and greater genetic response similar to GS with model updating (Müller et al. 2017, 2018).

While TS's had relatively small impacts on response metrics, they were highly significant. Since relatedness of TS's and validation sets affect estimates of prediction accuracy it has been suggested that model updating needs to be evaluated for accuracies of prediction within and across populations (Crossa et al. 2014; Juliana et al. 2018; Stewart-Brown et al. 2019). Herein, the TS's were comprised of a combined population of RIL's derived from all families across cycles of selection. Given a constant number of RILs in the TS's, within or across family prediction accuracies will depend on genetic differentiation among families (de Roos et al. 2009; Schulz-Streeck et al. 2012; Stewart-Brown et al. 2019). However, actual soybean breeding projects evaluate a few to many dozen RIL's per family and future simulation studies, especially 
688

689

690

691

692

693

694

695

696

697

698

699

700

701

702

703

704

705

706

707

708

709

710

of two part systems, should consider whether relationships between evaluation units, RIL's and selection units (Holland et al. 2003), possibly individuals, need to be used in design of the TS's.

We allowed the size of TS's to increase every cycle by adding data from prior cycles. Increasing the number of RIL's per TS requires more computational resources. An alternative strategy is to randomly sample subsets of data from each of the prior cycles to maintain a constant cumulative training population size. It is also possible to assign weights to the samples from prior cycles to place more weight on data from more recent cycles. These possibilities suggest determination of an optimal combination of numbers of RIL's and weights that will provide maximum prediction accuracy with minimal computational requirements. Some aspects of this optimization problem have been addressed (Lorenz et al. 2013; Hickey et al. 2014; Akdemir et al. 2015; Xavier et al. 2017). For example, Akdemir et al (2015) devised a genetic algorithm for selecting optimal training populations to minimize prediction error variance and Xavier et al (2017) developed sampling methods for training Bayesian GP models. Another consideration is whether TS's need to be updated every cycle. Instead of updating the model with data from every cycle would it be more effective to retrain GP models every second third, fourth... cycle while maintaining a constant training population size? Modifications to design of TS's will need to be addressed with simulations before implementation.

Like most simulation studies we fixed levels of SI as constant for all combinations of factors across all cycles of recurrent selection. This is not consistent with actual soybean breeding projects. Just as sampling families result in some with exceptional genotypes and some with poor genotypes, in an actual genetic improvement project there is variability among cycles. The effects of applying a dynamic selection strategy is an alternative and interesting question. We hypothesize that a strategy consisting of applying different SI's, optimized for each cycle, will 
711 achieve improved long-term genetic response by differentially emphasizing genetic variance and

712 genetic gain across multiple cycles of selection.

713 Unlike actual soybean genetic improvement projects we simulated a closed breeding population

714 derived from a sample of SoyNAM founders in which culled lines were not resampled for

715 discarded favorable alleles, nor did we simulate exchange of lines among breeding projects. In

716 MZ's II, III and IV there are six public breeding projects and about a dozen commercial soybean

717 breeding projects. Thus, there is potential, depending on material transfer agreements, to

718 exchange genotypes among breeding populations. In our next set of investigations migration

719 among multiple breeding projects will be evaluated for response to recurrent selection within and

720 among breeding projects using island model evolutionary algorithms (Hagan et al. 2012; Yabe et

721 al. 2016). Results reported herein will provide comparators for assessing impacts of migration

722 policies relative to the other factors that affect responses to RGS.

723 Also, as with prior simulation studies, we simulated truncation selection, but unlike previous

724 investigations we did not randomly mate selected lines each cycle. Rather, we used the hub

725 network design (Guo et al. 2013; Guo et al. 2014). We did not consider relationships among

726 selected RILs nor the trade-offs between genetic gain, genetic variance (inbreeding) when

727 selecting RILs to cross. There exist multi-objective optimization methods such as genomic

728 mating and optimal cross selection (Rutten et al. 2002; Woolliams et al. 2015; Akdemir and

729 Sánchez 2016; Gorjanc et al. 2018; Akdemir et al. 2019) that have been demonstrated to provide

730 both greater rates of genetic gain and assure maintenance of population genetic potential across

731 cycles.

732 In the future, it is possible that development of male sterile and insect pollination systems for

733 soybean (Ortiz-Perez 2008; Davis 2020) will enable a cycle of RGS to be conducted using two or 
734 three mating generations per year. This will enable an alternative genetic improvement system

735 based on decoupling genetic improvement from variety development (Gaynor et al. 2017). By

736 separating the two types of breeding projects, GS can be applied continuously. In the two part

737 system TS's will need to be composed of genotypic and phenotypic data obtained from annual

738 field trials of RIL's, although the TS's will be several selection cycles removed from the cycle

739 used to create the RILs.

740 Results reported herein suggest that RGS in a two part system will rapidly produce genetic gains

741 and loss of useful genetic variance in very short periods of time. Indeed, 40 cycles of RGS could

742 be completed in 12 to 20 years. To offset the shorter cycle times, application of GP models to

743 select and cross individual progeny instead of RIL's could result in a larger effective population

744 size per cycle by creating more opportunities for recombination and slow the unintended loss of

745 valuable alleles in discarded linkage blocks. However, before investments in development of

746 male sterile and insect pollination systems for soybean (Ortiz-Perez 2008; Davis, personal

747 communication) research using simulations are needed to understand the trade-offs and whether

748 the investments are justified using approaches from Operations Research (Xu et al. 2011;

749 Cameron et al. 2017, Han et al. 2017).

751 Acknowledgements

752 Funding for this research was provided by the Department of Agronomy, Iowa State University,

753 the North Central Soybean Research Program and an NSF grant (1830478). Supplemental

754 funding for large scale computing was enabled by the Extreme Science and Engineering

755 Discovery Environment (XSEDE), which is supported by National Science Foundation. XSEDE 
resources consisted of research allocations (DMS190015 \& DMS190018) on PSC-Bridges Large Memory nodes for the simulations involving updating of parametric GP models. The Iowa State University -Pronto GPU cluster enabled computation of SVM model update simulations. We want to acknowledge Matheus de Krause for discussions on fitting non-linear models using 'nlme' package, Dr. Lizhi Wang for efficient programs to simulate meiosis and Dr. Alencar Xavier for sharing an efficient expectation maximization method for fitting ridge regression GP models. Last, we want to thank anonymous critical reviewers of earlier versions of this manuscript for providing useful suggestions on how to present such a large volume of information from such a comprehensive investigation of expected short and long term outcomes of GS applied to soybeans adapted to MZ's II to IV.

\section{Reference}

Achard F, Butruille M, Madjarac S, Nelson PT, Duesing J, Laffont JL, Nelson B, Xiong J, Mikel MA, Smith JSC: Single nucleotide polymorphisms facilitate dus testing of soybean cultivars for plant variety protection. Crop Science 2020, n/a.

Ahrent DK, Caviness CE: Natural Cross-Pollination of Twelve Soybean Cultivars in Arkansas. Crop Science 1994, 34:376-378.

Akdemir D, Beavis W, Fritsche-Neto R, Singh AK, Isidro-Sánchez J: Multi-objective optimized genomic breeding strategies for sustainable food improvement. Heredity 2019, 122:672-683.

Akdemir D, Sánchez JI: Efficient Breeding by Genomic Mating. Frontiers in Genetics 2016, 7. Akdemir D, Sanchez JI, Jannink JL: Optimization of genomic selection training populations with a genetic algorithm. Genet Sel Evol 2015, 47:38.

Anderson EJ, Ali ML, Beavis WD, Chen P, Clemente TE, Diers BW, Graef GL, Grassini P, Hyten DL, McHale LK, et al: Soybean [Glycine max (L.) Merr.] Breeding: History, Improvement, Production and Future Opportunities. In Advances in Plant Breeding 
Strategies: Legumes: Volume 7. Edited by Al-Khayri JM, Jain SM, Johnson DV. Cham: Springer International Publishing; 2019: 431-516

Asoro FG, Newell MA, Beavis WD, Scott MP, Jannink J-L: Accuracy and Training Population Design for Genomic Selection on Quantitative Traits in Elite North American Oats. Iowa State University Digital Repository; 2011.

Bassi FM, Bentley AR, Charmet G, Ortiz R, Crossa J: Breeding schemes for the implementation of genomic selection in wheat (Triticum spp.). Plant Science 2016, 242:2336. response to genomic selection: effects of estimation method and reference population structure for different genetic architectures. Genetics Selection Evolution 2012, 44:3. 5 (2015) 2015.

Beavis WD: The power and deceit of QTL experiments: lessons from comparative QTL studies. In Proceedings of the forty-ninth annual corn and sorghum industry research conference. Chicago, IL; 1994: 250-266.

Bernardo R: Molecular Markers and Selection for Complex Traits in Plants: Learning from the Last 20 Years. Crop Science 2008, 48:1649.

Beyene Y, Semagn K, Mugo S, Tarekegne A, Babu R, Meisel B, Sehabiague P, Makumbi D, Magorokosho $\mathrm{C}$, Oikeh $\mathrm{S}$, et al: Genetic gains in grain yield through genomic selection in eight bi-parental maize populations under drought stress.(RESEARCH)(Author abstract). 2015, 55:154.

Bijma P: Long-term genomic improvement - new challenges for population genetics. Journal of Animal Breeding and Genetics 2012, 129:1-2.

Brisbane J, Gibson J: Balancing selection response and rate of inbreeding by including genetic relationships in selection decisions. International Journal of Plant Breeding Research 1995, 91:421-431.

Brooks C, Nekrasov V, Lippman ZB, Van Eck J: Efficient gene editing in tomato in the first generation using the clustered regularly interspaced short palindromic repeats/CRISPRassociated9 system. Plant Physiol 2014, 166:1292-1297. 
Bulmer MG: The Effect of Selection on Genetic Variability. The American Naturalist 1971, 105:201-211.

Bulmer MG: The effect of selection on genetic variability: a simulation study. Genet Res 1976, 28:101-117.

Byrum J, Beavis B, Davis C, Doonan G, Doubler T, Kaster V, Mowers R, Parry S: Genetic Gain Performance Metric Accelerates Agricultural Productivity. Interfaces 2017, 47:442-453.

Cameron JN, Han Y, Wang L, Beavis WD: Systematic design for trait introgression projects. Theoretical and Applied Genetics 2017, 130:1993-2004.

Cannon SB, Shoemaker RC: Evolutionary and comparative analyses of the soybean genome. Breeding science 2012, 61:437-444.

Carlson JB, Lersten NR: Reproductive Morphology. In Soybeans: Improvement, Production, and Uses. 2004:59-95.

Carter Jr TE, Nelson RL, Sneller CH, Cui Z: Genetic diversity in soybean. Soybeans:

Improvement, production, and uses 2004, 16:303-416.

Caviness CE: Estimates of Natural Crosspollination in Jackson Soybeans in Arkansas1. Crop Science 1966, 6:cropsci1966.0011183X000600020034x.

Cockerham CC, Burrows PM: Selection limits and strategies. Proceedings of the National Academy of Sciences of the United States of America 1980, 77:546.

Collins LM, Dziak JJ, Kugler KC, Trail JB: Factorial Experiments: Efficient Tools for Evaluation of Intervention Components: Efficient Tools for Evaluation of Intervention Components. American Journal of Preventive Medicine 2014, 47:498-504.

Cooper M, Podlich D, Micallef K, Smith O, Jensen N, Chapman S, Kruger N: Complexity, quantitative traits and plant breeding: a role for simulation modelling in the genetic improvement of crops. Quantitative genetics, genomics and plant breeding'(Ed MS Kang) pp 2002:143-166.

Crossa J, Pérez P, Hickey J, Burgueño J, Ornella L, Cerón-Rojas J, Zhang X, Dreisigacker S, Babu R, Li Y, et al: Genomic prediction in CIMMYT maize and wheat breeding programs. Heredity 2014, 112:48-60.

Daetwyler HD, Calus MPL, Pong-Wong R, de Los Campos G, Hickey JM: Genomic prediction in animals and plants: simulation of data, validation, reporting, and benchmarking. Genetics 2013, 193:347-365.

De Los Campos G, Gianola D, Rosa GJM, Weigel KA, Crossa J: Semi-parametric genomicenabled prediction of genetic values using reproducing kernel Hilbert spaces methods. Genetics Research 2010, 92:295-308. 
de los Campos G, Hickey JM, Pong-Wong R, Daetwyler HD, Calus MPL: Whole-Genome Regression and Prediction Methods Applied to Plant and Animal Breeding. Genetics 2013, 193:327-345.

de los Campos G, Naya H, Gianola D, Crossa J, Legarra A, Manfredi E, Weigel K, Cotes JM: Predicting Quantitative Traits With Regression Models for Dense Molecular Markers and Pedigree. Genetics 2009, 182:375-385.

de Los Campos G, Vazquez AI, Fernando R, Klimentidis YC, Sorensen D: Prediction of complex human traits using the genomic best linear unbiased predictor. PLoS Genet 2013, 9:e1003608.

de Roos APW, Hayes BJ, Goddard ME: Reliability of genomic predictions across multiple populations. Genetics 2009, 183:1545-1553.

Dempfle L: A note on increasing the limit of selection through selection within families. Genet Res 1974, 24:127-135.

Diers BW, Specht J, Rainey KM, Cregan P, Song Q, Ramasubramanian V, Graef G, Nelson R, Schapaugh W, Wang D, et al: Genetic Architecture of Soybean Yield and Agronomic Traits. G3: Genes|Genomes|Genetics 2018, 8:3367.

\section{Dunn K: Process Improvement using Data. In.; 2015}

Emily C, Rex B: Accuracy of Genomewide Selection for Different Traits with Constant Population Size, Heritability, and Number of Markers. The Plant Genome 2013, 6.

Endelman JB: Ridge Regression and Other Kernels for Genomic Selection with R Package rrBLUP. The Plant Genome Journal 2011, 4:250.

Fehr W: Principles of cultivar development: theory and technique. Macmillian Publishing Company; 1991.

Fehr WR, Hadley HH: Hybridization of crop plants. American Society of Agronomy and Crop Science Society of America; 1980.

Forsberg SK, Bloom JS, Sadhu MJ, Kruglyak L, Carlborg Ö: Accounting for genetic interactions improves modeling of individual quantitative trait phenotypes in yeast. Nat Genet 2017, 49:497-503.

Garber RJ, Odland T, McIlvaine T, Quisenberry K: Varietal Experiments with Soybeans. Agricultural Experiment Station, College of Agriculture, West Virginia ...; 1925.

Gaynor RC, Gorjanc G, Bentley AR, Ober ES, Howell P, Jackson R, Mackay IJ, Hickey JM: A Two-Part Strategy for Using Genomic Selection to Develop Inbred Lines. Crop Science 2017, 57:2372-2386.

Goddard M: Genomic selection: prediction of accuracy and maximisation of long term 
response. Genetica 2009, 136:245-257.

Goiffon M, Kusmec A, Wang L, Hu G, Schnable PS: Improving Response in Genomic

Goldberg S: Introduction to difference equations, with illustrative examples from economics, psychology, and sociology. New York: New York, Wiley; 1958.

Göring HH, Terwilliger JD, Blangero J: Large upward bias in estimation of locus-specific effects from genomewide scans. The American Journal of Human Genetics 2001, 69:13578941369.

Gorjanc G, Gaynor RC, Hickey JM: Optimal cross selection for long-term genetic gain in two-part programs with rapid recurrent genomic selection. Theoretical and Applied Genetics 2018, 131:1953-1966.

GOUDET J: hierfstat, a package for $\mathbf{r}$ to compute and test hierarchical F-statistics.

Grant D, Cregan P, Shoemaker RC: Genome organization in dicots: Genome duplication in

Grant D, Nelson RT, Cannon SB, Shoemaker RC: SoyBase, the USDA-ARS soybean genetics and genomics database. Nucleic acids research 2010, 38:D843.

Guo Z, Tucker DM, Basten CJ, Gandhi H, Ersoz E, Guo B, Xu Z, Wang D, Gay G: The impact of population structure on genomic prediction in stratified populations. Theoretical and Applied Genetics 2014, 127:749-762.

Guo Z, Tucker DM, Lu J, Kishore V, Gay G: Evaluation of genome-wide selection efficiency in maize nested association mapping populations. Theoretical and Applied Genetics 2012,

915 Guzman C, Peña RJ, Singh R, Autrique E, Dreisigacker S, Crossa J, Rutkoski J, Poland J, 916 Battenfield S: Wheat quality improvement at CIMMYT and the use of genomic selection on 917 it. Applied \& Translational Genomics 2016, 11:3-8.

918 Habier D, Fernando RL, Dekkers JCM: The Impact of Genetic Relationship Information on 
Hagan S, Knowles J, Kell DB: Exploiting Genomic Knowledge in Optimising Molecular Breeding Programmes: Algorithms from Evolutionary Computing (Evolutionary Computing for Molecular Breeding). 2012, 7:e48862.

Han Y, Cameron JN, Wang L, Beavis WD: The Predicted Cross Value for Genetic Introgression of Multiple Alleles. Genetics 2017, 205:1409.

Hayes BJ, Visscher PM, Goddard ME: Increased accuracy of artificial selection by using the realized relationship matrix. Genetics Research 2009, 91:47-60.

Heffner EL, Sorrells ME, Jannink J-L: Genomic Selection for Crop Improvement. Crop Science 2009, 49:1.

Heslot N, Akdemir D, Sorrells ME, Jannink J-L: Integrating environmental covariates and crop modeling into the genomic selection framework to predict genotype by environment interactions. Theoretical and Applied Genetics 2014, 127:463-480.

Heslot N, Jannink J-L, Sorrells ME: Perspectives for Genomic Selection Applications and Research in Plants. Crop Science 2015, 55:1-12.

Heslot N, Yang H-P, Sorrells ME, Jannink J-L: Genomic Selection in Plant Breeding: A Comparison of Models. Crop Science 2012, 52:146-160.

Hickey JM, Chiurugwi T, Mackay I, Powell W: Genomic prediction unifies animal and plant breeding programs to form platforms for biological discovery. Nature genetics 2017, 49:1297.

Hickey JM, Dreisigacker S, Crossa J, Hearne S, Babu R, Prasanna BM, Grondona M, Zambelli A, Windhausen VS, Mathews K, Gorjanc G: Evaluation of Genomic Selection Training Population Designs and Genotyping Strategies in Plant Breeding Programs Using Simulation. Crop Science 2014, 54:1476-1488.

Hickey JM, Gorjanc G: Simulated data for genomic selection and genome-wide association studies using a combination of coalescent and gene drop methods. G3 (Bethesda, Md) 2012, 2:425.

Hill WG, Robertson A: The effect of linkage on limits to artificial selection. Genetics Research 2008, 89:311-336. (First published in 1968)

Holland J, Nyquist WE, Cervantes-Martinez CT: Estimating and interpreting heritability for plant breeding: Anupdate. Plant breeding reviews 2003, 22:9-111.

Hou J, van Leeuwen J, Andrews BJ, Boone C: Genetic Network Complexity Shapes Background-Dependent Phenotypic Expression. Trends in genetics : TIG 2018, 34:578-586.

Howard R, Carriquiry AL, Beavis WD: Parametric and nonparametric statistical methods for genomic selection of traits with additive and epistatic genetic architectures. $G 3$ (Bethesda) 2014, 4:1027-1046. 
Howard R, Carriquiry AL, Beavis WD: Application of Response Surface Methods To Determine Conditions for Optimal Genomic Prediction. G3: Genes|Genomes|Genetics 2017, 7:3103.

Hung HY, Browne C, Guill K, Coles N, Eller M, Garcia A, Lepak N, Melia-Hancock S, Oropeza-Rosas M, Salvo S, et al: The relationship between parental genetic or phenotypic divergence and progeny variation in the maize nested association mapping population. Heredity (Edinb) 2012, 108:490-499.

Hyten DL, Song Q, Zhu Y, Choi IY, Nelson RL, Costa JM, Specht JE, Shoemaker RC, Cregan PB: Impacts of genetic bottlenecks on soybean genome diversity. Proc Natl Acad Sci U S A 2006, 103:16666-16671.

Jannink J-L: Dynamics of long-term genomic selection. Genetics Selection Evolution 2010, 42:35.

Jannink JL, Lorenz AJ, Iwata H: Genomic selection in plant breeding: from theory to practice. Brief Funct Genomics 2010, 9:166-177.

Jenko J, Gorjanc G, Cleveland MA, Varshney RK, Whitelaw CBA, Woolliams JA, Hickey JM: Potential of promotion of alleles by genome editing to improve quantitative traits in livestock breeding programs. Genetics Selection Evolution 2015, 47:55.

Jombart T: adegenet: a $\mathbf{R}$ package for the multivariate analysis of genetic markers. Bioinformatics 2008, 24:1403-1405.

Jombart T, Ahmed I: adegenet 1.3-1: new tools for the analysis of genome-wide SNP data. Bioinformatics 2011, 27:3070-3071.

Jonas E, de Koning D-J: Does genomic selection have a future in plant breeding? Trends in Biotechnology 2013, 31:497-504.

Jonas E, de Koning DJ: Goals and hurdles for a successful implementation of genomic selection in breeding programme for selected annual and perennial crops. Biotechnology \&amp; genetic engineering reviews 2016, 32:18.

Juliana P, Singh RP, Poland J, Mondal S, Crossa J, Montesinos-López OA, Dreisigacker S, Pérez-Rodríguez P, Huerta-Espino J, Crespo-Herrera L, Govindan V: Prospects and Challenges of Applied Genomic Selection-A New Paradigm in Breeding for Grain Yield in Bread Wheat. The Plant Genome 2018, 11:180017.

Kang H: Limits of artificial selection under balanced mating systems. 1979.

Kang H: Limits of artificial selection under balanced mating systems with family selection. Silvae genetica 1983, 32:188-195.

Kang H, Namkoong G: Limits of artificial selection under unbalanced mating systems. Theoretical and Applied Genetics 1980, 58:181-191. 
Kang H, Nienstaedt H: Managing long-term tree breeding stock. Silvae genetica 1987, 36:3039.

Kurek A: "Phenotypic and genomic selection for multi-trait improvement in soybean line and variety development" 2018.

Langewisch T, Lenis J, Jiang G-L, Wang D, Pantalone V, Bilyeu K: The development and use of a molecular model for soybean maturity groups. BMC Plant Biology 2017, 17:91.

Lemmon ZH, Reem NT, Dalrymple J, Soyk S, Swartwood KE, Rodriguez-Leal D, Van Eck J, Lippman ZB: Rapid improvement of domestication traits in an orphan crop by genome editing. Nat Plants 2018, 4:766-770.

Li Y, Kadarmideen HN, Dekkers JCM: Selection on multiple QTL with control of gene diversity and inbreeding for long-term benefit. Journal of Animal Breeding and Genetics 2008, 125:320-329.

Liu H, Meuwissen TH, Sorensen AC, Berg P: Upweighting rare favourable alleles increases long-term genetic gain in genomic selection programs. Genet Sel Evol 2015, 47:19.

Long N, Gianola D, Rosa GJM, Weigel KA: Application of support vector regression to genome-assisted prediction of quantitative traits. Theoretical and Applied Genetics 2011, 123: 1065 .

Long N, Gianola D, Rosa GJM, Weigel KA, Kranis A, GonzÁLez-Recio O: Radial basis function regression methods for predicting quantitative traits using SNP markers. Genetics Research 2010, 92:209-225.

Lorenz AJ: Resource Allocation for Maximizing Prediction Accuracy and Genetic Gain of Genomic Selection in Plant Breeding: A Simulation Experiment. G3: Genes|Genomes|Genetics 2013, 3:481.

Marulanda J, Mi X, Melchinger A, Xu J-L, Würschum T, Longin C: Optimum breeding strategies using genomic selection for hybrid breeding in wheat, maize, rye, barley, rice and triticale. Theor Appl Genet 2016, 129:1901-1913.

McCouch S, Baute GJ, Bradeen J, Bramel P, Bretting PK, Buckler E, Burke JM, Charest D, Cloutier S, Cole G: Feeding the future. Nature 2013, 499:23-24.

Meuwissen T, Hayes B, Goddard M: Prediction of total genetic value using genome-wide dense marker maps. Genetics 2001, 157:1819-1829.

Meuwissen T, Hayes B, Goddard M: Accelerating improvement of livestock with genomic selection. Annu Rev Anim Biosci 2013, 1:221-237.

Meuwissen TH: Maximizing the response of selection with a predefined rate of inbreeding. Journal of animal science 1997, 75:934-940. 
Meuwissen TH, Goddard ME: Mapping multiple QTL using linkage disequilibrium and linkage analysis information and multitrait data. Genetics Selection Evolution 2004, 36:261.

Michel S, Ametz C, Gungor H, Epure D, Grausgruber H, Loschenberger F, Buerstmayr H:

Montesinos-López OA, Martín-Vallejo J, Crossa J, Gianola D, Hernández-Suárez CM,

Müller D, Schopp P, Melchinger AE: Persistency of Prediction Accuracy and Genetic Gain in Synthetic Populations Under Recurrent Genomic Selection. G3: Genes|Genomes|Genetics 2017, 7:801.

Myers RH: Response surface methodology / Raymond H. Myers. s.1. : [Ann Arbor: s.1. : s.n. Ann

Nakaya A, Isobe SN: Will genomic selection be a practical method for plant breeding? Genes|Genomes|Genetics 2018. an example. Ecology and evolution 2019, 9:10225-10240.

1051 Orf JH: 2 - Breeding, Genetics, and Production of Soybeans. In Soybeans. Edited by Johnson LA, White PJ, Galloway R: AOCS Press; 2008: 33-65

Ortiz-Perez E, Mian RMA, Cooper RL, Mendiola T, Tew J, Horner HT, Hanlin SJ, Palmer RG: Seed-set evaluation of four male-sterile, female-fertile soybean lines using alfalfa leafcutting bees and honey bees as pollinators. The Journal of Agricultural Science 2008, 146:461-469.

1057 Peccoud J, Velden KV, Podlich D, Winkler C, Arthur L, Cooper M: The selective values of alleles in a molecular network model are context dependent. Genetics 2004, 166:1715-1725. 
Ryman N, Leimar O: GST is still a useful measure of genetic differentiation - a comment on Jost's D. Molecular Ecology 2009, 18:2084-2087.

1077 Schulz-Streeck T, Ogutu JO, Karaman Z, Knaak C, Piepho HP: Genomic Selection using 1078 Multiple Populations. Crop Science 2012, 52:2453-2461. 1996, 144:329-338.

Song Q, Hyten DL, Jia G, Quigley CV, Fickus EW, Nelson RL, Cregan PB: Fingerprinting Hyten D, Nelson R, et al: Genetic Characterization of the Soybean Nested Association Mapping Population. The Plant Genome 2017, 10. in major US field crops 2014, 33:311-355. 
Weir BS: Disequilibrium. methods for discrete population genetic data 1996:91-139.

Wilcox JR, Schapaugh Jr. WT, Bernard RL, Cooper RL, Fehr WR, Niehaus MH: Genetic

Selection. Genetics 2013, 195:573.

Woolliams J, Corbin L: Coalescence theory in livestock breeding. Journal of Animal Breeding optimization. J Anim Breed Genet 2015, 132:89-99.

Xavier A: Efficient Estimation of Marker Effects in Plant Breeding. G3: Bioinformatics 2017, 18:191.

Xavier A, Thapa R, Muir WM, Rainey KM: Population and quantitative genomic properties of the USDA soybean germplasm collection. Plant Genetic Resources 2018:1-11. in Soybeans. G3: Genes|Genomes|Genetics 2016, 6:2611.

1117 Xu S: Theoretical basis of the Beavis effect. Genetics 2003, 165:2259-2268.

$1118 \mathrm{Xu}$ P, Wang L, Beavis WD: An optimization approach to gene stacking. European Journal of 1119 Operational Research 2011, 214:168-178.

1120 Yabe S, Yamasaki M, Ebana K, Hayashi T, Iwata H: Island-Model Genomic Selection for 
bioRxiv preprint doi: https://doi.org/10.1101/2020.02 14.949008; this version posted October 30,2020. The copyright holder for this preprint

(which was not certified by peer review) is the author/funder, who has granted bioRxiv a license to display the preprint in perpetuity. It is made available under aCC-BY 4.0 International license.

1127 Zuur A: Mixed Effects Models and Extensions in Ecology with R by Alain Zuur, Elena N. Ieno, 1128 Neil Walker, Anatoly A. Saveliev, Graham M. Smith. 1st ed. 2009.. edn: New York, NY :

1129 Springer New York : Imprint: Springer; 2009.

1130

1131

1132

1133 


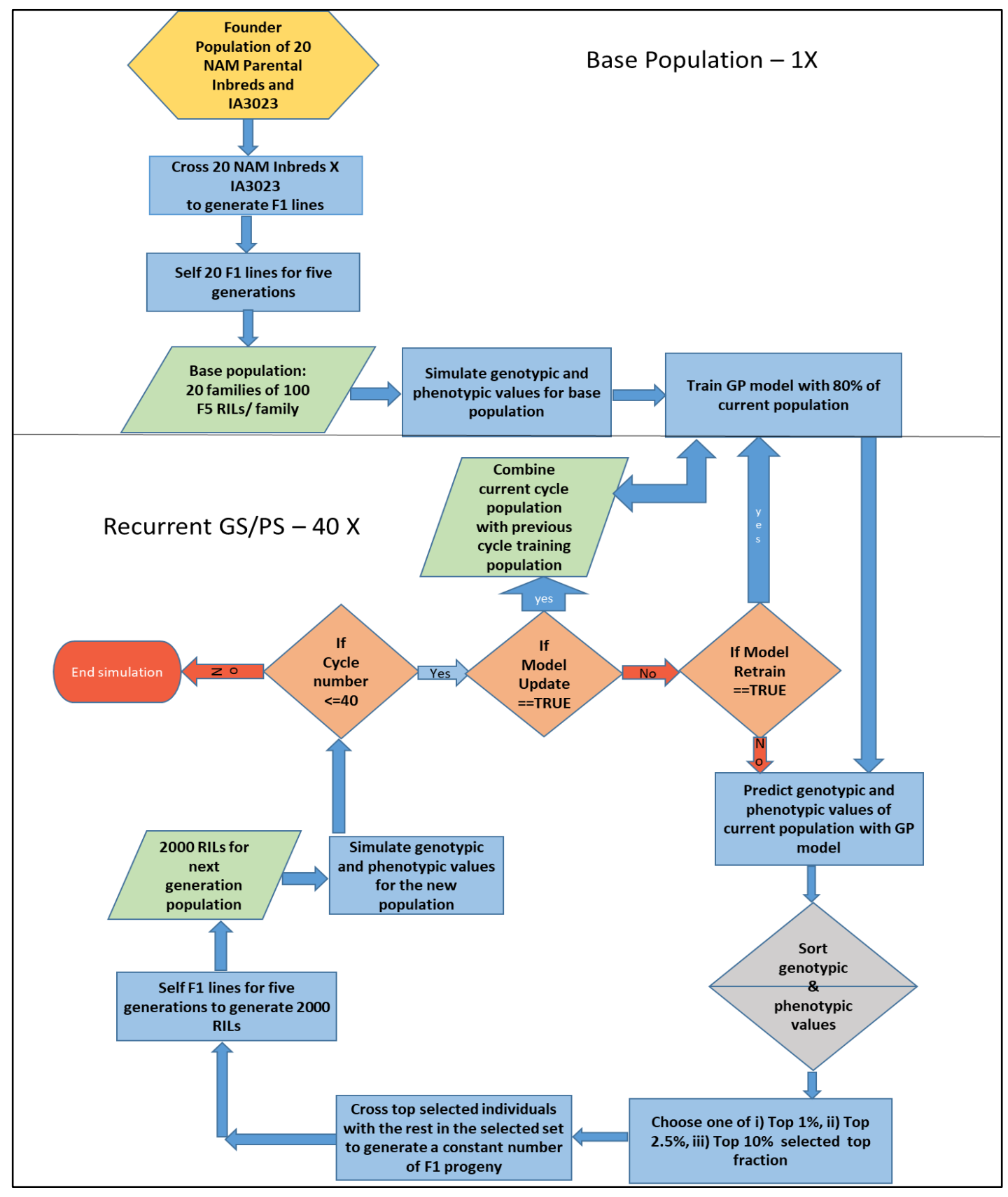

Figure 1 Flow Chart for Simulations of Recurrent Genomic Selection. The upper half panel represents the steps involved in generating the base population of $2000 \mathrm{~F}_{5}$ RILs derived from 20 NAM founder lines crossed, in silico, to IA3023. It includes the model training step for genomic prediction models. The lower half panel represents recurrent steps of prediction, sorting, truncation selection, crossing, and generation of $F_{5}$ RILs for each cycle as well as the decision steps to check if the training set should be updated and if the recurrent process is to be continued for another cycle. 


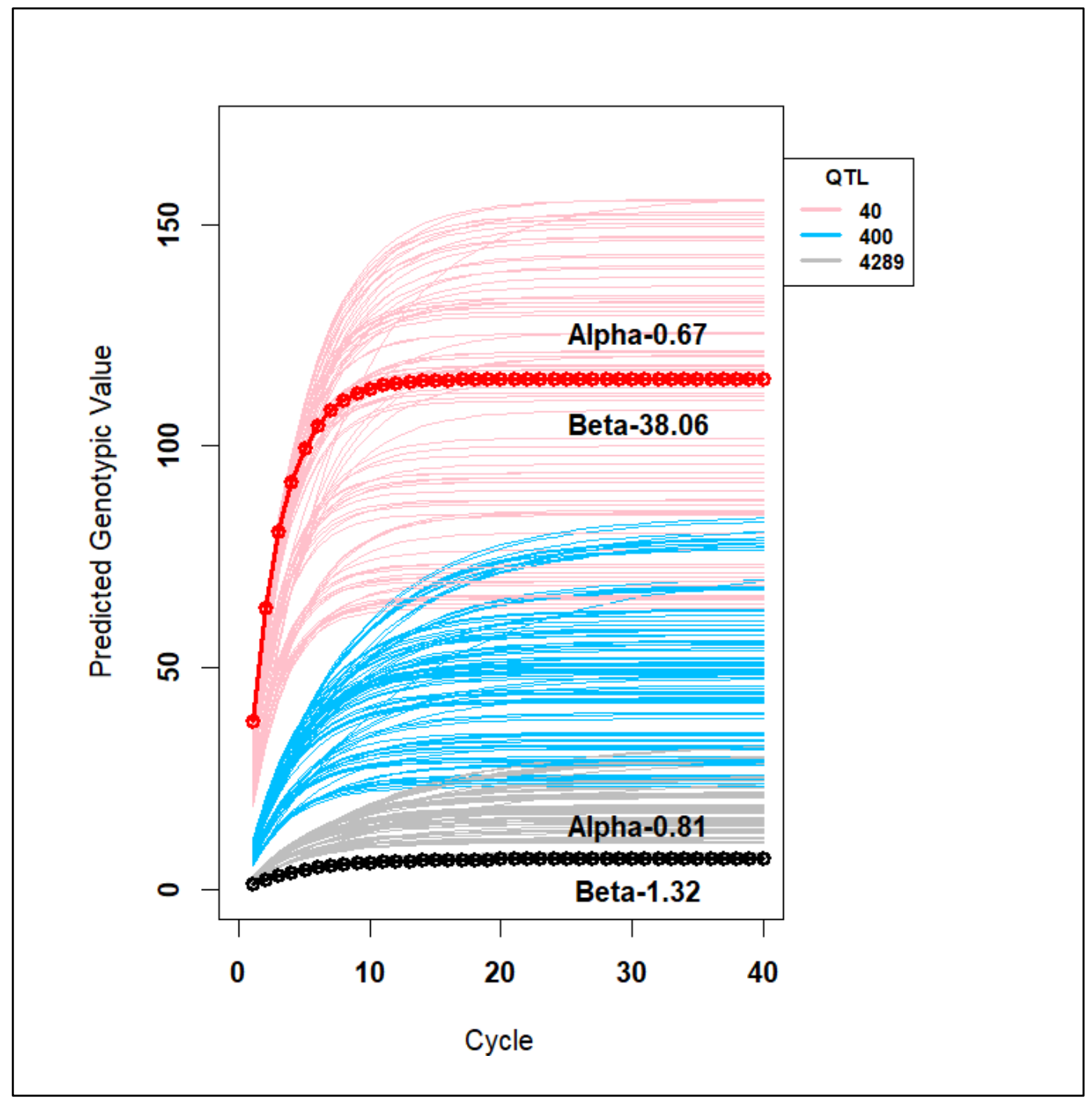

Figure 2 Theoretical Genotypic Values: Theoretical Genotypic Values from 40 cycles of recurrent selection modeled with the recurrence equation, where $y_{c}$ represents the genotypic value in cycle $c$, with $c=1,2 \ldots 40$ and values of $\alpha$ and $\beta$ range from $0.6-0.9$ and $1.32-38.06$ respectively for 360 combinations of factors across all selection methods, training sets, selection intensities, number of simulated QTL and simulated heritabilities. The bold lines represent curves with the smallest and largest beta values and their corresponding alpha values. The curves are colored corresponding to 40 (pink), 400 (blue) and 4289 (gray) simulated QTL. 


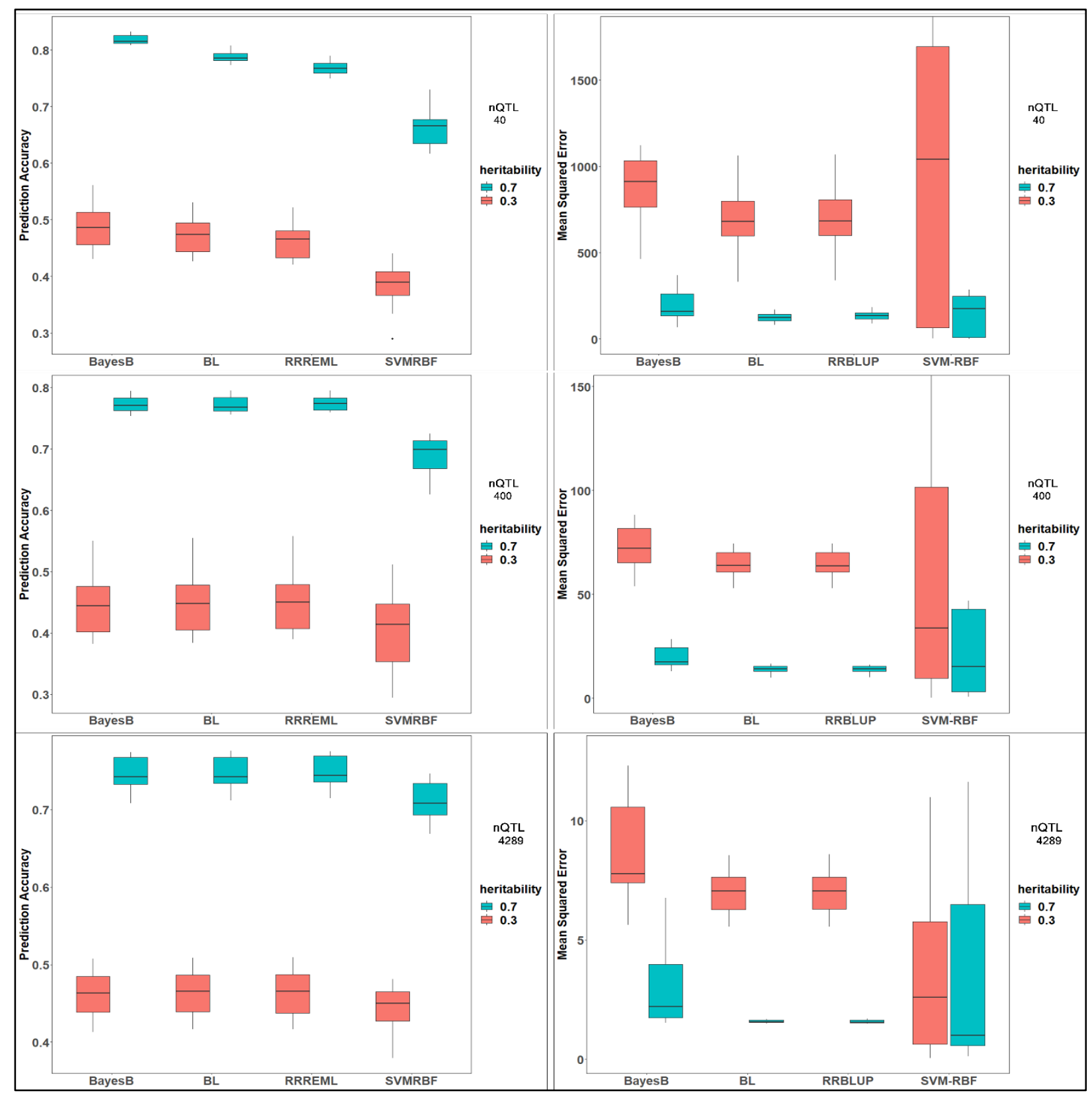

Figure 3 Estimated prediction accuracies and MSE in Founding Set of RILs: Estimated prediction accuracies (left panel) and mean squared errors (right panel) for four genomic prediction (GP) models: BayesB, BL (Bayes LASSO), RRREML (Ridge Regression with REML) and SVMRBF (Support Vector Machines with Radial Basis Function Kernel) trained with $F_{5}$ RILs derived from crosses of 20 homozygous founder lines with IA3023. Phenotypes used to train the GP models consisted of genetic architectures comprised of 40,400 and 4289 simulated QTL (top, middle and bottom) that were responsible for $70 \%$ (blue) and $30 \%$ (red) of phenotypic variability in the initial populations. 
bioRxiv preprint doi: https://doi.org/10.1101/2020.02 14.949008; this version posted October 30,2020 . The copyright holder for this preprint (which was not certified by peer review) is the author/funder, who has granted bioRxiv a license to display the preprint in perpetuity. It is made available under aCC-BY 4.0 International license.

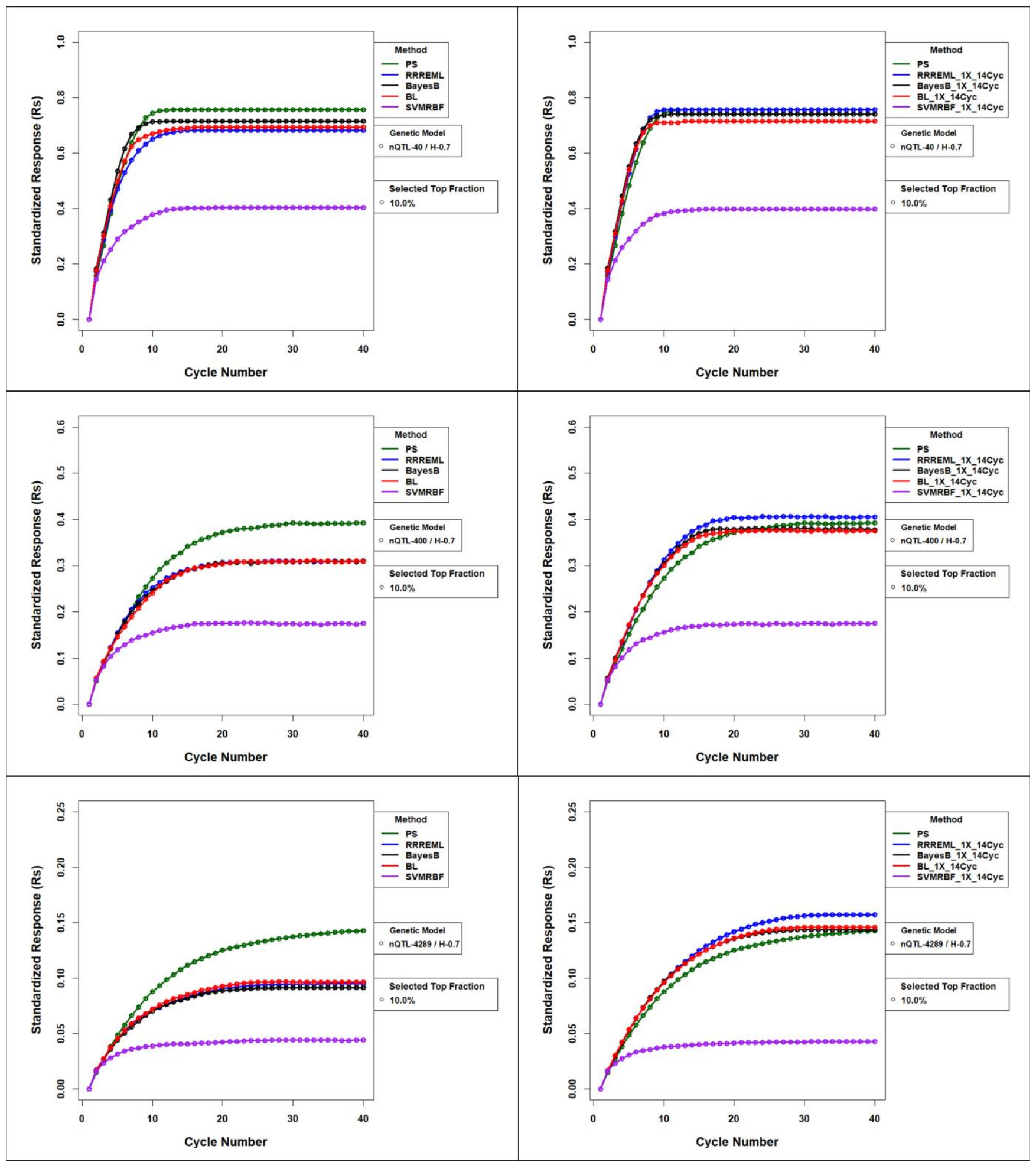


Figure 4 Standardized Responses for Comparison of GS methods with and without Updating for $0.7 \mathrm{H}$ and Top $10 \%$ Selected Fraction Forty cycles of standardized responses to selection of $10 \%$ of 2000 soybean RILs per cycle. Standardized responses are plotted by selection methods without (left panels) and with (right panels) model updating using prior cycles as training sets for the four genotypic prediction models. Phenotypic selection (PS) is not updated and hence is the same in the left and right panels. The top panels consist of responses for genetic architectures consisting of 40 simulated QTL. Middle panels consist of responses for genetic architectures consisting of 400 simulated QTL and the bottom panels consist of responses for genetic architectures consisting of 4289 simulated QTL. All 40, 400, and 4289 simulated QTL are responsible for $70 \%$ of phenotypic variability in the initial population. PS - Phenotypic Selection, RR-REML- Ridge Regression with Restricted Maximum Likelihood, BL - Bayes LASSO, and SVMRBF- Support Vector Machine with Radial Basis Kernel. 


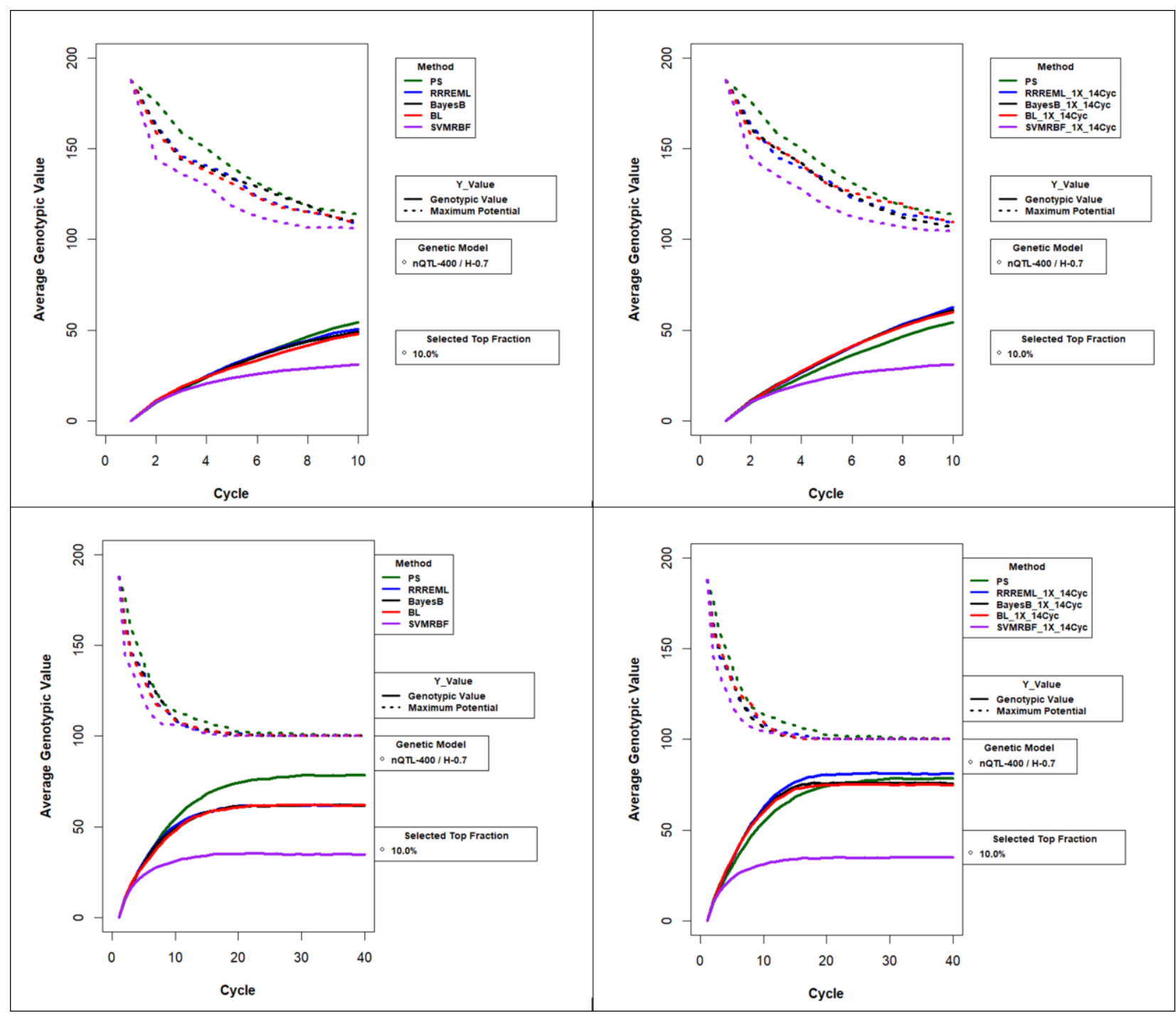

Figure 5 Average Genotypic Value and Maximum Genetic Potential for Comparison of GS methods with and without Updating for $0.7 \mathrm{H}$ and Top $10 \%$ Selected Fraction Average genotypic value and maximum possible genotypic value in recurrent selection of $10 \%$ of 2000 soybean RILs per cycle. The values are plotted by selection methods without (left panels) and with (right panels) model updating using prior cycles as training sets for the four genotypic prediction models. Plots demonstrate decrease in maximum possible genotypic value due to loss of favorable alleles and increase in average genotypic value for 10 cycles (upper panel) and 40 cycles (lower panel) of selection. Phenotypic selection (PS) is not updated and hence is the same in the left and right panels. The top and bottom panels represent genotypic values for genetic architectures consisting of 400 simulated QTL responsible for $70 \%$ of phenotypic variability in the initial population. PS - Phenotypic Selection, RR-REML- Ridge Regression with Restricted Maximum Likelihood, BL - Bayes LASSO, and SVMRBF- Support Vector Machine with Radial Basis Kernel. 


\begin{tabular}{|c|c|c|c|c|c|c|c|c|c|c|}
\hline Method & QTL & heritability & TH5 & TH10 & TH15 & TH20 & TH25 & TH30 & TH35 & TH40 \\
\hline RR-REML & 40 & 0.7 & -2.36 & -12.71 & -9.63 & -9.61 & -9.61 & -9.61 & -9.61 & -9.61 \\
\hline BayesB & 40 & 0.7 & 10.96 & -4.18 & -5.49 & -5.49 & -5.49 & -5.49 & -5.49 & -5.49 \\
\hline BL & 40 & 0.7 & 3.46 & -9.96 & -8.77 & -8.24 & -8.24 & -8.24 & -8.24 & -8.24 \\
\hline SVMRBF & 40 & 0.7 & -39.67 & -49.09 & -46.90 & -46.67 & -46.67 & -46.67 & -46.67 & -46.67 \\
\hline & & & & & & & & & & \\
\hline RR-REML & 400 & 0.7 & 2.42 & -7.37 & -14.57 & -17.25 & -19.61 & -20.96 & -20.93 & -21.11 \\
\hline BayesB & 400 & 0.7 & 0.10 & -9.53 & -14.89 & -17.58 & -19.94 & -21.26 & -21.05 & -21.11 \\
\hline BL & 400 & 0.7 & -3.35 & -11.95 & -15.15 & -18.26 & -19.62 & -21.21 & -20.80 & -21.37 \\
\hline SVMRBF & 400 & 0.7 & -21.91 & -43.06 & -49.93 & -52.95 & -54.26 & -55.48 & -55.46 & -55.42 \\
\hline & & & & & & & & & & \\
\hline RR-REML & 4289 & 0.7 & -6.26 & -20.12 & -25.33 & -27.60 & -29.16 & -30.98 & -32.60 & -33.57 \\
\hline BayesB & 4289 & 0.7 & -9.07 & -19.79 & -26.28 & -29.14 & -31.30 & -33.37 & -34.90 & -35.84 \\
\hline BL & 4289 & 0.7 & -5.85 & -17.94 & -23.50 & -25.70 & -27.24 & -29.66 & -31.36 & -32.35 \\
\hline SVMRBF & 4289 & 0.7 & -34.49 & -55.74 & -63.38 & -66.01 & -66.85 & -67.79 & -68.60 & -69.15 \\
\hline
\end{tabular}

Figure 6 Heat Map for Percent Gain in Rs Relative to PS for 0.7 H for GP Models without Updating: Heat map indicating standardized response relative to $P S$ as percentage gain after 40 cycles of recurrent selection using genomic prediction models without updated training sets for $40,400,4289$ simulated QTL responsible for $70 \%$ of phenotypic variability in the initial population. Blue to red shaded cells represent increasing gain in response relative to PS. RR-REML- Ridge Regression with Restricted Maximum Likelihood, BL Bayes LASSO, and SVMRBF- Support Vector Machine with Radial Basis Kernel. 


\begin{tabular}{|c|c|c|c|c|c|c|c|c|c|c|c|}
\hline Method & QTL & heritability & No. Prior Cycles & TH5 & TH10 & TH15 & TH20 & TH25 & TH30 & TH35 & TH40 \\
\hline & & & & & & & & & & & \\
\hline RR-REML & 40 & 0.7 & 14 & 9.04 & 1.55 & 0.00 & 0.00 & 0.00 & 0.00 & 0.00 & 0.00 \\
\hline BayesB & 40 & 0.7 & 14 & 14.41 & -1.05 & -2.06 & -2.06 & -2.06 & -2.06 & -2.06 & -2.06 \\
\hline BL & 40 & 0.7 & 14 & 11.89 & -4.70 & -5.49 & -5.49 & -5.49 & -5.49 & -5.49 & -5.49 \\
\hline SVMRBF & 40 & 0.7 & 14 & -39.85 & -48.58 & -47.63 & -47.36 & -47.36 & -47.36 & -47.36 & -47.36 \\
\hline & & & & & & & & & & & \\
\hline RR-REML & 400 & 0.7 & 14 & 11.34 & 14.84 & 12.30 & 8.71 & 5.73 & 3.29 & 3.50 & 3.22 \\
\hline BayesB & 400 & 0.7 & 14 & 13.05 & 12.00 & 8.38 & 1.75 & -1.11 & -2.96 & -3.02 & -3.91 \\
\hline BL & 400 & 0.7 & 14 & 13.85 & 10.44 & 6.45 & 1.02 & -1.89 & -4.24 & -3.92 & -4.55 \\
\hline SVMRBF & 400 & 0.7 & 14 & -21.66 & -42.81 & -50.39 & -53.52 & -54.86 & -55.38 & -55.48 & -55.40 \\
\hline & & & & & & & & & & & \\
\hline RR-REML & 4289 & 0.7 & 14 & 10.01 & 10.51 & 11.79 & 13.39 & 14.52 & 13.67 & 11.70 & 10.13 \\
\hline BayesB & 4289 & 0.7 & 14 & 10.10 & 10.41 & 9.09 & 8.31 & 7.20 & 4.56 & 2.06 & 0.60 \\
\hline BL & 4289 & 0.7 & 14 & 10.32 & 9.22 & 8.87 & 8.78 & 8.22 & 6.11 & 3.59 & 2.10 \\
\hline SVMRBF & 4289 & 0.7 & 14 & -36.73 & -56.79 & -64.13 & -66.87 & -68.08 & -68.97 & -69.49 & -69.96 \\
\hline
\end{tabular}

Figure 7 Heat Map for Percent Gain in Rs Relative to PS for 0.7 H for GP Models with Updating: Heat map indicating standardized response relative to PS as percentage gain after 40 cycles of recurrent selection using genomic prediction models with updated training sets from up to 14 prior cycles of selection for 40,400 , and 4289 simulated QTL responsible for $70 \%$ of phenotypic variability in the initial population. Blue to red shaded cells represent increasing gain in response relative to PS. RR-REML- Ridge Regression with Restricted Maximum Likelihood, BL - Bayes LASSO, and SVMRBF- Support Vector Machine with Radial Basis Kernel. 
bioRxiv preprint doi: https://doi.org/10.1101/2020.02 14 949008; this version posted October 302020 . The copyright holder for this preprint (which was not certified by peer review) is the author/funder, who has granted bioRxiv a license to display the preprint in perpetuity. It is made available under aCC-BY 4.0 International license.

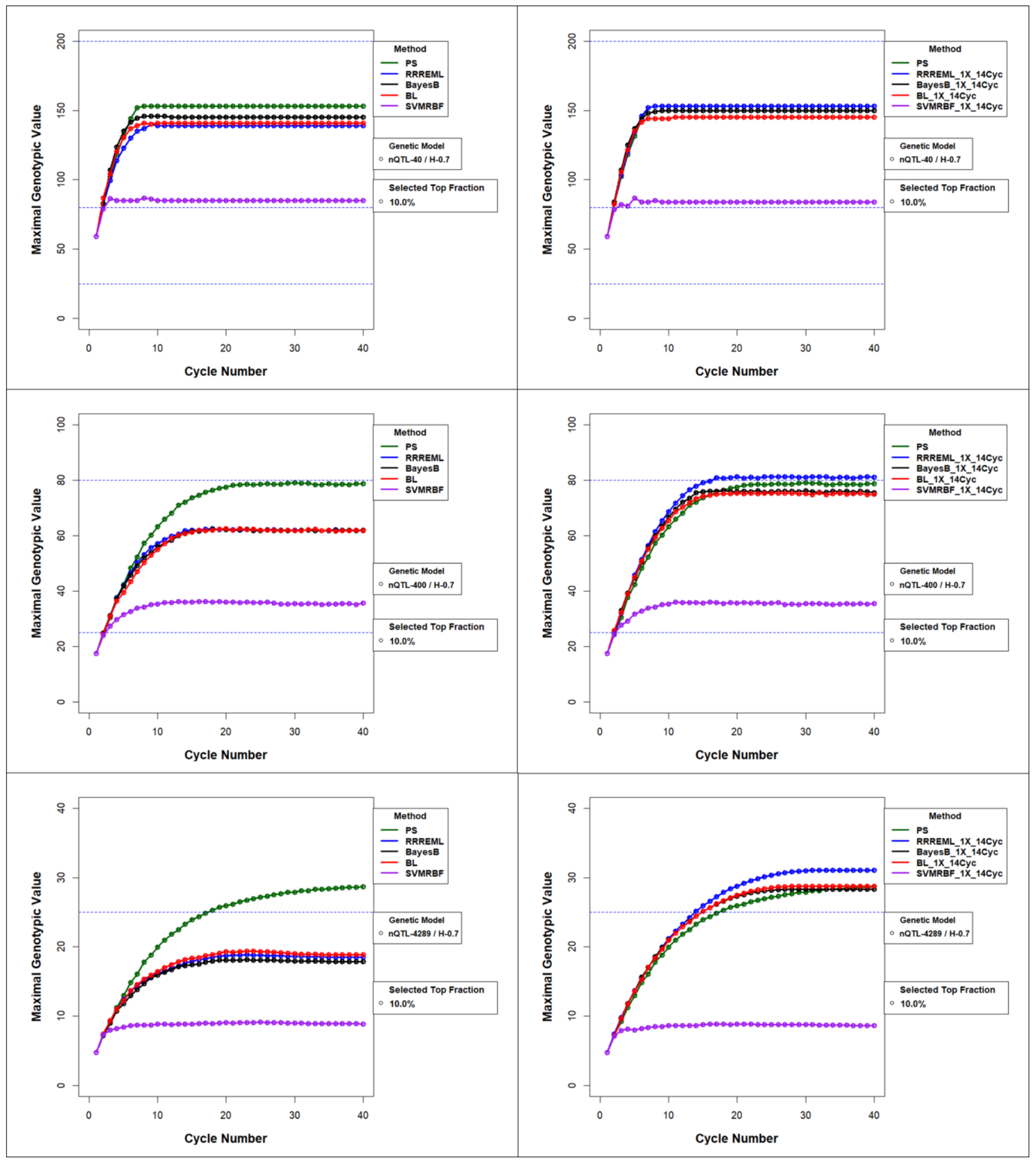


Figure 8 Maximal Genotypic Value for Comparison of GS methods with and without Updating for 0.7 $\mathrm{H}$ and Top 10\% Selected Fraction: Maximum attained genotypic values (Mgvs) in recurrent genomic selection and phenotypic selection (PS) without updating the training sets in the left panels and with training set updates from up to 14 prior cycles in the right panels. PS has no training sets and hence does not change between the left and right panels. a) 40 QTL (top), b) 400 QTL (middle) and c) 4289 QTL (bottom) responsible for $70 \%$ of phenotypic variability in the initial population and selection of $10 \%$ of the RILs in each cycle. PS - Phenotypic Selection, RR-REML- Ridge Regression with Restricted Maximum Likelihood, BL - Bayes LASSO, and SVMRBF- Support Vector Machine with Radial Basis Kernel. 
bioRxiv preprint doi: https://doi.org/10.1101/2020.02.14.949008; this version posted October 30,2020. The copyright holder for this preprint (which was not certified by peer review) is the author/funder, who has granted bioRxiv a license to display the preprint in perpetuity. It is made available under aCC-BY 4.0 International license.

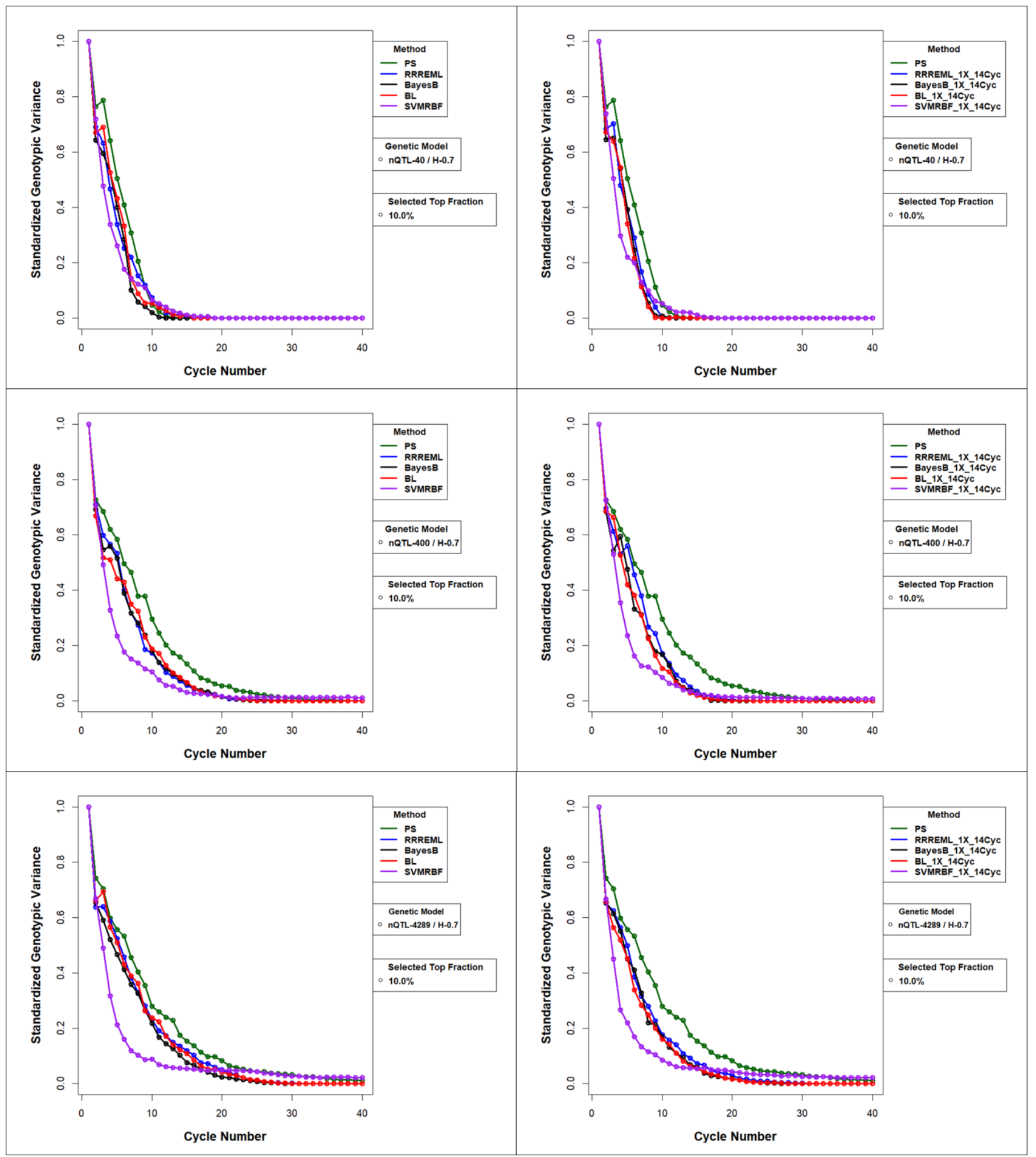


Figure 9 Standardized Genotypic Variance (Sgv) for Comparison of GS methods with and without Updating for $0.7 \mathrm{H}$ and Top $10 \%$ Selected Fraction Standardized genotypic variance without training set updating (left panels) and with training set updating using prior cycle training data (right panels) for the four GP models. PS has no updating and hence is the same in both left and right panels. A) Training data from up to 14 prior cycles for 40 simulated QTL (top), 400 simulated QTL (middle) and 4289 simulated QTL (bottom) responsible for $70 \%$ of phenotypic variability in the initial population and top $10 \%$ of RILs with the greatest predicted values. PS - Phenotypic Selection, RR-REML- Ridge Regression with Restricted Maximum Likelihood, BL - Bayes LASSO, and SVMRBF- Support Vector Machine with Radial Basis Kernel. 


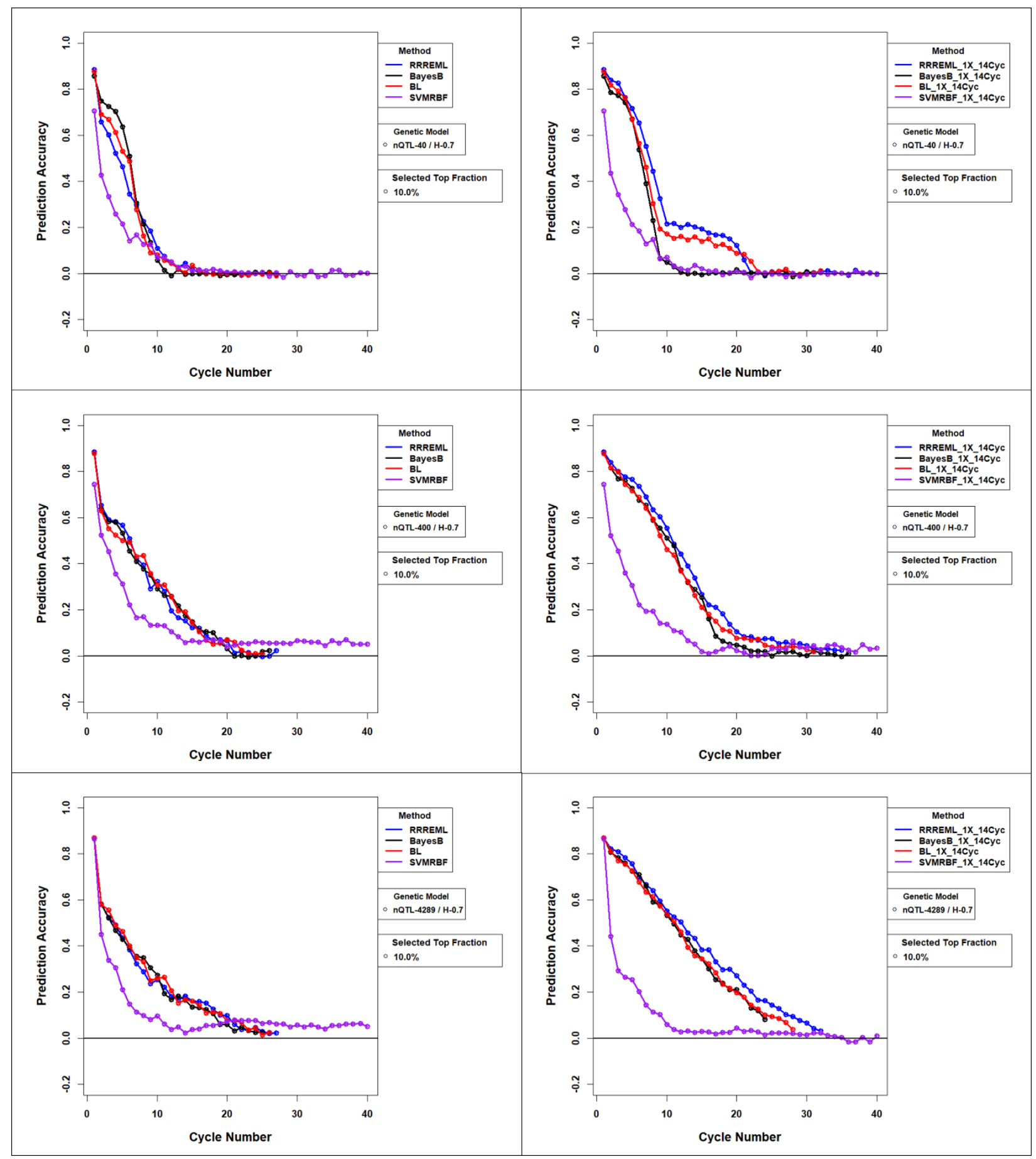

Figure 10 Estimated Prediction Accuracies for Comparison of GS methods with and without Updating for $0.7 \mathrm{H}$ and Top $10 \%$ Selected Fraction: Estimated prediction accuracies with updates to the training sets used in genomic prediction (GP) models. Training data from up to 14 prior selection cycles were used to update all four GP models for 40 QTL (top), 400 QTL (middle) and 4289 QTL (bottom) responsible for $70 \%$ of phenotypic variability in the initial population and top $10 \%$ of RILs with the greatest predicted values. RR-REML- Ridge Regression with Restricted Maximum Likelihood, BL Bayes LASSO, and SVMRBF- Support Vector Machine with Radial Basis Kernel. 


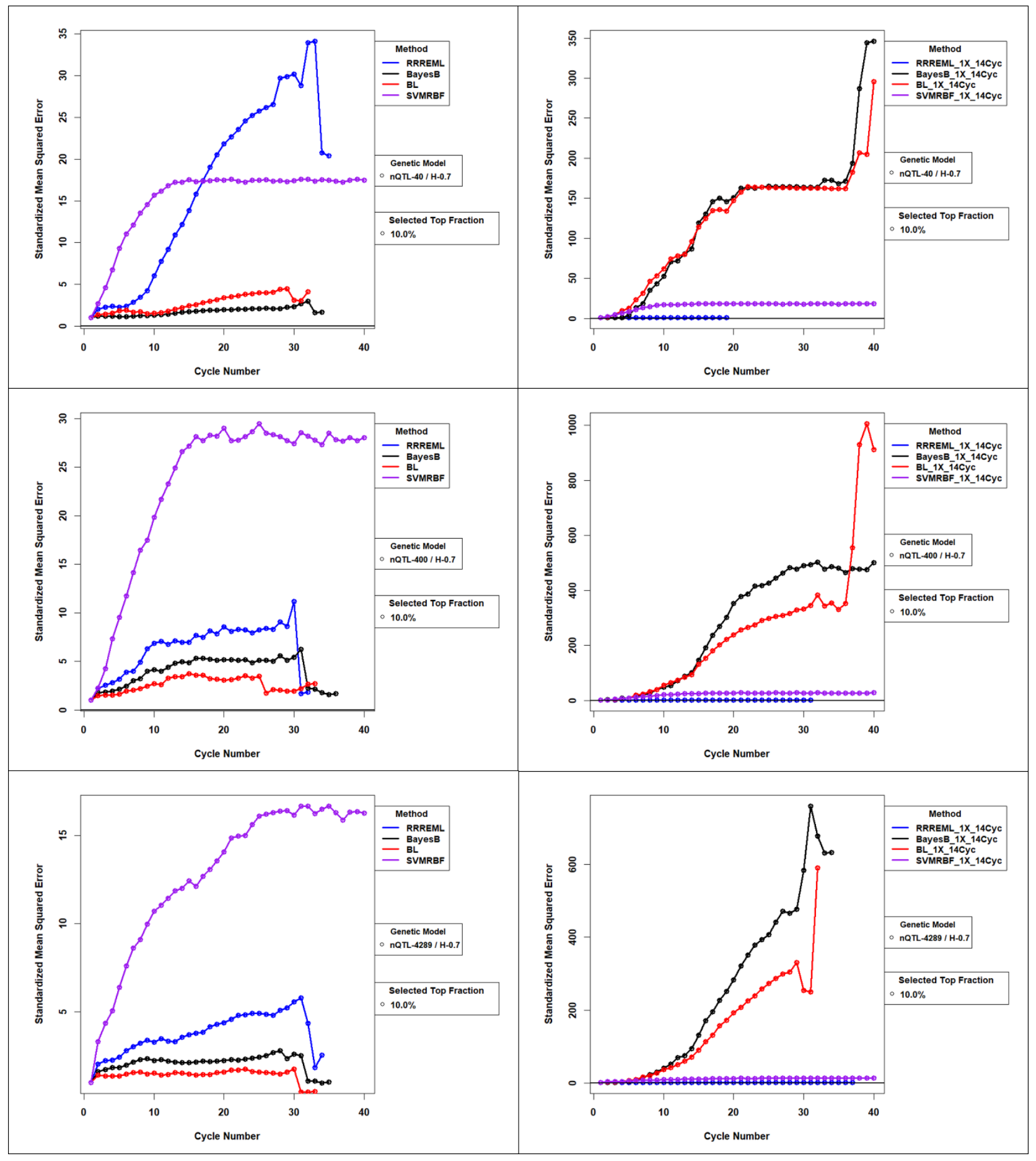

Figure 11 Standardized Mean Squared Error for Comparison of GS methods with and without Updating for $0.7 \mathrm{H}$ and Top 10\% Selected Fraction: Mean Squared Error of GP models with updates to the training sets used in genomic prediction (GP) models. Standardized MSE $(>=1)$ is estimated as the ratio of MSE for GP models in cycle 'c' to MSE for GP models trained with founder population of RILs. While MSE for RRREML model were lesser with model updating, MSE for bayesian methods increased orders of magnitude in late cycles of selection with model updating. SVMEBF showed relatively constant MSE with and without updating. Training data from up to 14 prior selection cycles were used to update all four GP models for 40 QTL (top), 400 QTL (middle) and 4289 QTL (bottom) responsible for $70 \%$ of phenotypic variability in the initial population and top $10 \%$ of RILs with the greatest predicted values. RR-REML- Ridge Regression with Restricted Maximum Likelihood, BL - Bayes LASSO, and SVMRBF- Support Vector Machine with Radial Basis Kernel. 
TABLE 1: FACTORIAL DESIGN

\begin{tabular}{|c|c|c|}
\hline Factors & Number of Levels & Values for Levels \\
\hline Number of QTL & 3 & $40,400,4289$ \\
\hline Heritability & 2 & $0.7,0.3$ \\
\hline Selection Intensity & 3 & $2.67,2.34,1.75$ \\
\hline Selection Method & 5 & $\begin{array}{l}\text { i) PS- Phenotypic value } \\
\text { ii) GS - GP(RR-REML) } \\
\text { iii) GS - GP(Bayes B) } \\
\text { iv) GS - GP(Bayes LASSO) } \\
\text { v) GS - GP(SVM, Radial basis } \\
\text { Function Kernel) }\end{array}$ \\
\hline $\begin{array}{l}\text { Model Update: number } \\
\text { of prior cycles used in } \\
\text { training sets }\end{array}$ & 4 & $\begin{array}{l}\text { i) } 0 \text { previous cycles } \\
\text { ii) } 10 \text { previous cycles } \\
\text { iii) } 12 \text { previous cycles } \\
\text { iv) } 14 \text { previous cycles }\end{array}$ \\
\hline $\begin{array}{c}\text { Total Number of unique } \\
\text { combinations }\end{array}$ & 360 & \\
\hline $\begin{array}{l}\text { Total Number of } \\
\text { Simulations }\end{array}$ & $\begin{array}{l}3600 \text { with } 10 \text { reps } \\
\text { /condition }\end{array}$ & \\
\hline
\end{tabular}




\section{TABLE 2 PACKAGES IN R FOR PARAMETRIC AND NON-PARAMETRIC MODELS WITH TUNING PARAMETERS}

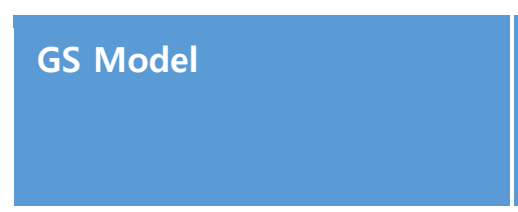

Ridge Regression

Bayesian LASSO

Bayes B

SVM

\section{Package (R)}

BGLR (Perez et al. 2014 )

BGLR (Perez et al. 2014 )

Rgtsvm (Wang et al. 2017) 2019)

\section{Model Tuning Parameters \\ (BGLR package )}

REML-EM (custom R script Xavier

EM algorithm for estimation of parameters with REML method without using matrix inversion

Priors for

$\operatorname{varE}(\mathrm{df}=3, \mathrm{~S}=0.25) ; \operatorname{var} U$

$(d f=3, S=0.63)$;

lambda $($ shape $=0.53$, rate $=5 e-5)$

type='random',value $=30$ ),

nlter $=20000$,

burnln=2000,

thin $=1$

nlter $=41000$, burnln $=1000$,

$\mathrm{dfO}=4, \mathrm{R} 2=0.7$

SVM with Radial basis function kernel on GPU 